\section{1}

\title{
The Enterprise: A massive transposon carrying Spok meiotic drive genes
}

\author{
Aaron A. Vogan*1, S. Lorena Ament-Velásquez ${ }^{1}$, Eric Bastiaans ${ }^{1,2}$, Ola Wallerman ${ }^{3}$, Sven J. \\ Saupe $^{4}$, Alexander Suh ${ }^{1,5}$, Hanna Johannesson ${ }^{1}$ \\ ${ }^{1}$ Systematic Biology, Department of Organismal Biology, Uppsala University, Norbyvägen 18D, 75236 \\ Uppsala, Sweden \\ ${ }^{2}$ Laboratory of Genetics, Wageningen University, Arboretumlaan 4, 6703 BD, Wageningen, The \\ Netherlands \\ ${ }^{3}$ Department of Medical Biochemistry and Microbiology, Comparative genetics and functional genomics; \\ Uppsala University, Husargatan 3, 75237 Uppsala, Sweden \\ ${ }^{4}$ IBGC, UMR 5095, CNRS Université de Bordeaux, 1 rue Camille Saint Saëns, 33077 Bordeaux, cedex, \\ France \\ ${ }^{5}$ Present address: School of Biological Sciences, University of East Anglia, Norwich Research Park, NR4 \\ 7TU, Norwich, United Kingdom \\ *For Correspondence: aaron.vogan@ebc.uu.se
}

\section{Abstract}

The genomes of eukaryotes are full of parasitic sequences known as transposable elements (TEs). Most TEs studied to date are relatively small (50 - $12000 \mathrm{bp}$ ), but can contribute to very large proportions of genomes. Here we report the discovery of a giant tyrosine-recombinasemobilized DNA transposon, Enterprise, from the model fungus Podospora anserina. Previously, we described a large genomic feature called the Spok block which is notable due to the presence of meiotic drive genes of the Spok gene family. The Spok block ranges from $110 \mathrm{~kb}$ to $247 \mathrm{~kb}$ and can be present in at least four different genomic locations within $P$. anserina, despite what is an otherwise highly conserved genome structure. We have determined that the reason for its varying positions is that the Spok block is not only capable of meiotic drive, but is also capable of transposition. More precisely, the Spok block represents a unique case where the Enterprise has captured the Spoks, thereby parasitizing a resident genomic parasite to become a genomic hyperparasite. Furthermore, we demonstrate that Enterprise (without the Spoks) is found in other fungal lineages, where it can be as large as $70 \mathrm{~kb}$. Lastly, we provide experimental evidence that the Spok block is deleterious, with detrimental effects on spore production in strains which carry it. In contrast to the selfish role of the Enterprise in $P$. anserina, we hypothesize that the mobility of the Enterprise may also play an adaptive role in fungi when Enterprise undergoes horizontal transfer while carrying metabolic genes. This union of meiotic drivers and a transposon has created a selfish element of impressive size in Podospora, challenging our perception of how TEs influence genome evolution and broadening the horizons in terms of what the upper limit of transposition may be. 


\section{Introduction}

Transposable elements (TEs) are major agents of change in eukaryotic genomes. Their ability to selfishly parasitise their host replication machinery has large impacts on both genome size and on gene regulation (Chénais et al. 2012). In extreme cases, TEs can contribute up to $85 \%$ of genomic content (Schnable et al. 2009) and expansion and reduction of TEs can result in rapid changes in both genome size and architecture (Haas et al. 2009; Talla et al. 2017; Möller and Stukenbrock 2017). Generally, TEs have small sizes ( 50 - $12000 \mathrm{bp}$ ) and accomplish these large-scale changes through their sheer number. For example, there are $\sim 1.1$ million Alu elements in the human genome, which have had a large impact on genome evolution (Bennett et al. 2008; Jurka 2004). The largest known cases among Class I retrotransposons are long terminal repeat (LTR) elements that can be as large as $30 \mathrm{~kb}$, but among Class II DNA transposons, Mavericks/Polintons are known to grow as large as $40 \mathrm{~kb}$ through the capture of additional open reading frames (ORFs) (Arkhipova and Yushenova 2019). Recently, a behemoth TE named Teratorn was described in teleost fish; it can be up to $182 \mathrm{~kb}$ in length, dwarfing all other known TEs. Teratorn has achieved this impressive size by fusing a piggyBac DNA transposon with a herpesvirus, thereby blurring the line between TEs and viruses (Inoue et al. 2017, 2018). Truly massive transposons may be lurking in the depths of many eukaryotic genomes, but the limitations of short-read genome sequencing technologies and the lack of population-level high-quality assemblies may make them difficult to identify.

The Spok block is a large genomic feature that was first identified thanks to the presence of the spore killing (Spok) genes in species from the genus Podospora (Vogan, AmentVelásquez, et al. 2019; Grognet et al. 2014). The Spoks are selfish genetic elements that bias their transmission to the next generation in a process known as meiotic drive. Here, drive occurs by inducing the death of spores that do not inherit them, through a single protein that operates as both a toxin and an antidote (Vogan, Ament-Velásquez, et al. 2019; Grognet et al. 2014). The first Spok gene described, Spok1, was discovered in P. comata (Grognet et al. 2014). In P. anserina, the homologous gene Spok2 is found at high population frequencies, while two other genes of the Spok family, Spok3 and Spok4, are at low to intermediate frequencies (Vogan, Ament-Velásquez, et al. 2019). Unlike Spok1 and Spok2, however, Spok3 and Spok4 are always associated with a large genomic region (the Spok block). The Spok block can be located at different chromosomal locations in different individuals, but is never found more than once in natural strains. The number of Spok genes and the location of the Spok block (which carries Spok3, Spok4 or both) define the overall meiotic driver behavior of a given genome, which can be classified into the so-called Podospora spore killers or Psks (Vogan, Ament-Velásquez, et al. 2019; van der Gaag et al. 2000). The Spok block stands out not only because of its size, typically around $150 \mathrm{~kb}$, but also because there is otherwise high genome synteny among strains of $P$. anserina and with the related species $P$. comata and $P$. pauciseta (Vogan, Ament80 Velásquez, et al. 2019). 
The fact that the Spok block is found at unique genomic positions between otherwise highly similar strains is of prime interest as each novel Spok block position creates a unique meiotic drive type (Psk) due to the intricacies of meiosis in Podospora. In this study, we have determined that the reason the Spok block occurs at multiple genomic locations is that the Spok block itself is actually a unique version of the Enterprise, a novel DNA transposon that is mobilized by a tyrosine recombinase (YR). The Spok block represents an Enterprise that has captured meiotic drive genes and subsequently grown to a massive size through a gradual accumulation of DNA sequence. We find copies of Enterprise without meiotic drive genes in other fungal species that are at least $70 \mathrm{~kb}$, placing Enterprise among the largest known TEs, even when not harbouring the Spoks. The Spok block version of Enterprise not only represents the largest TE discovered to date (reaching $247 \mathrm{~kb}$ ), but is also capable of both transposition and meiotic drive. Furthermore, our data suggest that the Spok block is associated with fitness costs in $P$. anserina. This discovery drastically changes what we know about the size limits of transposition, and provides new insight into the role of massive TEs in eukaryotic genome evolution.

\section{Results}

\section{Newly isolated Podospora strains reveal a novel spore killer type}

We isolated and sequenced with Illumina HiSeq technology a strain of $P$. anserina (named Wa137) and two strains of $P$. comata (Wa131 and Wa139) collected in Wageningen, the Netherlands. The strains Wa137 and Wa139 were also sequenced using MinION Oxford Nanopore technology, achieving similar quality to published chromosome-level assemblies (Supplementary Table 1). Additionally, we included in our analyses all published long-read genomes of $P$. anserina (10 strains), $P$. pauciseta (one), and $P$. comata (one) (Espagne et al. 2008; Vogan, Ament-Velásquez, et al. 2019; Silar et al. 2019), which are mostly assembled at chromosome level, with respective genome sizes around $36 \mathrm{Mb}$ (Supplementary Table 1). Previous work demonstrated that the Spok block can be found at three unique chromosomal positions among characterized $P$. anserina strains, defining the killer types $P s k-1 / 5$ (with the Spok block located at chromosome 3), Psk-2 (right arm of chromosome 5) and Psk-7/8 (left arm of chromosome 5) (Vogan, Ament-Velásquez, et al. 2019) (Table 1). The newly isolated strain Wa137 was found to have a Spok block with the largest size yet reported $(247 \mathrm{~kb})$ and in a novel position (chromosome 1), conferring it a new spore killing phenotype that we named Psk9 (see Vogan, Ament-Velásquez, et al. (2019) for the principle of nomenclature). The published P. pauciseta genome has a Spok block in chromosome 4 (Psk-P1), but it seems to represent a fragmented version of the $P$. anserina blocks (Vogan, Ament-Velásquez, et al. 2019). Unlike $P$. anserina and $P$. pauciseta, the newly isolated strains of $P$. comata were found to have a single full-copy Spok gene (Spok1) which is not associated with any Spok block-like features, in agreement with the previously published reference genome of $P$. comata (Table 1) (Grognet et al. 2014; Silar et al. 2019). 


\section{The Spok block moves via transposition}

121 A number of mechanisms exist by which a sequence can move within a genome, including reciprocal translocation, ectopic recombination, and transposition (Mieczkowski, Lemoine, and Petes 2006). We can rule out reciprocal translocations in the case of the Spok block, as overall chromosome synteny is preserved (Vogan, Ament-Velásquez, et al. 2019). Ectopic recombination is often mediated by TEs, and transposition is, by definition, the process of TE mobility. In order to determine a candidate mode by which the Spok block moves, we examined the four unique Spok block insertion sites. This analysis revealed that no specific TE was present at the insertion site, but showed that six base pairs (RGGTAG) are always present and are repeated at the end of the Spok block (Figure 1A and B). This repeated sequence may represent a target site duplication (TSD), which is a hallmark of transposition mechanisms (Wicker et al. 2007). Additionally, the Psk-1 and Psk-9 Spok block insertion sites constitute a partially palindromic sequence ATACYT||AGGTAG (Figure 1B), a characteristic of some TE target sites (Linheiro and Bergman 2012). Together, this finding supports an explanation whereby the Spok block is mobilized like a TE via transposition.

The terminal sequences of TEs are often composed of structural features that are intrinsic to the transposition mechanism; LTRs for LTR retrotransposons and terminal inverted repeats (TIRs) for transposase-mobilized DNA transposons, for example (Wicker et al. 2007). These features can thus be used to determine the type of transposition or transposon underlying a given translocation. With this expectation, we examined the ends of the Spok block (which are very similar across all instances (Vogan, Ament-Velásquez, et al. 2019)), but found no such structural features. Curiously, the ends of the Spok block have been previously identified as two unclassified repetitive elements called bufo and schoutedenella (Espagne et al. 2008) (Figure 1B). We suspect that these unrelated elements actually represent the two ends of the Spok block (bufo representing the first 463 bp and schoutedenella representing the last $231 \mathrm{bp}$ ), and are found on their own in the genome as remains of previous, partially deleted, Spok block copies. To test this assumption, we mapped the location of all bufo and schoutedenella elements present in the Podospora genomes. We found that both elements can be identified throughout the chromosomes, with a tendency to locate in TE-rich areas in all species (Figure 1C and Supplementary Figure 1). In agreement with the postulate that the genomic copies of bufo and schoutedenella represent past Spok block insertions, at the Psk-7 insertion site there is $\sim 1200 \mathrm{bp}$ of sequence that is homologous to the beginning of the Spok block. This is nearly $700 \mathrm{bp}$ longer than what bufo alone represents, and further implies that the Psk-7 Spok block was inserted at the same site as a previous, now largely deleted copy. Together, these findings further support the idea that the Spok block inserts at the specific target site sequence RGGTAG which is duplicated during transposition (Figure 1B).

\section{The Spok block accumulates sequence from the genome}


identify candidate genes capable of transposing it. We annotated all genome assemblies using a modified version of the annotation pipeline in (Vogan, Ament-Velásquez, et al. 2019), relying on additional RNA-seq data, as well as an improved, manually curated repeat library (see Methods). The content of the different copies of the Spok block is largely overlapping (Vogan, Lorena Ament-Velásquez, et al. 2019), although the Wa137 Spok block has a large region of unique sequence resulting in a total size of $247 \mathrm{~kb}$ (Figure 1C and Supplementary Figure 2). Very few genes were predicted within the Spok block by this methodology (as evidenced by the dip in the orange track on the chr 1.2 scaffold; Figure 1C), so we manually annotated the Spok block of representative strains (Wa53, Wa28, Wa58, and Wa137), which identified numerous hypothetical protein-coding genes (e.g., 67 in the Wa137 Spok block), many of which appear to be pseudogenes or gene fragments. The blocks do not generally exhibit unusually high TE load. For example, the Spok block in Wa137 is only composed of $10.5 \%$ annotated TEs (compared to genome wide estimates of $3-6 \%$, Supplementary Table S1), nor do they appear to have a strong signature of repeat-induced point mutation (RIP). RIP is a process that operates in numerous fungi, including Podospora (Graïa et al. 2001), by specifically inducing C-to-T mutations of any repeated sequence within the genome (Selker et al. 1987; Cambareri et al. 1989) and results in a drop of GC proportion in repetitive elements. Such a GC-drop is clear in many regions of the Podospora genome, but is conspicuously absent within the Spok block (Vogan, Ament-Velásquez, et al. 2019). While some of the predicted genes had identifiable homologs within the $P$. anserina reference genome (orange features in Figure 1C), many are absent from the reference but have sequence similarity to genes from other fungi (purple features in Figure 1C). Of note, many of the predicted genes have potential roles in various metabolic pathways, like metal tolerance or antimicrobial resistance (Supplementary Table S2). It thus seems likely that the Spok block has grown to its large size, at least in part, by accumulating non-repetitive sequence from elsewhere within the genome. The accumulation does not appear to have been a single event, but was more likely many individual events, as the genomic homologs are found on all chromosomes (green links in Figure 1C).

\section{The Enterprise}

The gene annotation provided us with very few clues as to the agent(s) responsible for the translocation of the Spok block, so we turned to another approach. We hypothesize that the Spok block was a much smaller element in the past, and that such an element could be still transposing in the genomes of Podospora species. The closely related species $P$. comata possesses both bufo and schoutedenella repeats, and appears to have large areas of homology to the Spok block (Supplementary Figure 1B). We discovered that the P. comata strain Wa139 contains a $39 \mathrm{~kb}$ subtelomeric region on chromosome 5 (henceforth referred to as the Enterprise) that is nearly completely composed of TEs and sequence homologous to the Spok block (Supplementary Figure 2). The ends of the Enterprise are consistent with the Spok block (both bufo and schoutedenella are present) and it possesses the RGGTAG target site duplication (Supplementary Figure 3A). Enterprise is absent at the orthologous location in $P$. anserina and $P$. pauciseta, is much smaller in the other $P$. comata strain $\mathrm{T}_{\mathrm{D}}(5.5 \mathrm{~kb})$, and unassembled in Wa131. The other $P$. comata strains possess bufo and schoutedenella at this location, suggesting that this specific Enterprise insertion is now polymorphic for its state of 
degradation. However, it is difficult to fully recapitulate the history of the region due to the limited number of strains and the fact that Wa139 also has another schoutedenella copy at the insertion site that is absent in the other strains (Supplementary Figure 3A). Manual annotation revealed five putative genes within the Wa139 Enterprise, four of which are homologous to the genes within the Spok block and thus represent good candidates for involvement in the transposition of Enterprise and therefore the Spok block.

None of these four genes have a known function and none are Spok homologs. We examined their predicted protein domains, searched the $P$. anserina genomes for homologs, and scanned genomic databases for similar sequences in an attempt to discern whether they may be integral to the movement of the Enterprise (Supplementary Table 3). Only one of the genes is a likely candidate for enacting the transposition of the Enterprise. This gene is always present as the first ORF in the Spok block, but is degraded and interrupted by multiple TEs in Wa139. The ORF possesses multiple domains which may be characteristic of specific transposons, as determined by bioinformatic analysis (see Methods): a domain of unknown function called DUF3435, a zinc finger domain, and a predicted CHROMO domain (Figure 1D). Zinc finger domains are important for DNA binding, and CHROMO domains are implicated in histone binding and may allow TEs to be targeted to specific regions of the genome (Kordis 2005). There is strong overlap between the DUF3435 domain and tyrosine recombinases (YRs), a group of enzymes important for DNA integration of other selfish genetic elements known as Cryptons, parasitic plasmids, and bacteria (Kojima and Jurka 2011). YRs are extremely divergent, and there is little conserved sequence identity between this gene and known YRs, but importantly, YRs are known to have a catalytic tetrad consisting of an R-H-R-Y motif (Esposito and Scocca 1997), which is overlapping with the DUF3435 domain (Figure 1D \& Supplementary Figure 4). Given these results, we consider this gene to be a type of YR which is responsible for transposing Enterprise, and name it "spore killing related crypton" or Kirc.

From the evidence presented here, we propose that the Spok block and the homologous region of Wa139 represent copies of a previously unknown group of DNA transposons, which we name Enterprise. Cryptons are a type of DNA transposon defined only by the presence of a YR domain (Wicker et al. 2007) and may possess a TSD or not (Kojima and Jurka 2011), thus Enterprise can be classified as a novel group of Crypton. We define Enterprise as being composed of a YR-encoding gene homologous to Kirc, and possessing a TSD (Figure 1E). The Spok block therefore represents a specific version of Enterprise that contains Spok genes.

To confirm our hypothesis that Enterprise is capable of transposition and selfish replication, we mined fungal genomes available on JGI MycoCosm for homologous proteins of Kirc that are present in multiple copies within a single genome. We identified such a case in Melanconium sp. NRRL 54901. In this genome, a homolog of Kirc is found at the beginning of a $\sim 70 \mathrm{~kb}$ region that is present in four copies in the genome. Critically, this region is flanked by the same TSD as the Spok block and the target site is in its full palindromic context CTACCT||AGGTAG (Supplementary Figure 5). The only gene homologous with the Enterprise in Podospora is the Kirc homolog, thereby confirming that the minimal feature for transposition 
is the putative YR-encoding gene, Kirc, and classifying this region of Melanconium sp. as an

247 Enterprise.

\section{Kirc is wide-spread in filamentous fungi}

Given the fact that Enterprise is present in at least one species outside of Podospora, we queried GenBank with the sequence of Kirc using BLASTp to determine how widespread Enterprise is. We recovered a total of 481 protein hits, which were almost exclusively from within the Pezizomycotina, although putative homologs were identified from ten basidiomycete genomes as well. A phylogeny of a representative set of sequences shows that the relationships among the homologs do not follow the expected species phylogeny (Figure 2). Numerous species have multiple Kirc homologs in the same genome, some of which are not closely related. For example, within P. anserina, one homolog was recovered, Pa_5_10116 (see Espagne et al. (2008) for Podospora gene notation), which appears to be distantly related to Kirc, yet highly similar to homologs from Fusarium. Furthermore, Pa_5_10116 is pseudogenized and absent in the close relatives of $P$. anserina, $P$. comata and $P$. pauciseta, consistent with it being a transposable element. Attempts to describe TSDs in other fungi were largely unsuccessful as conserved flanks could not be identified in most cases, which is not unusual for Cryptons in general (Kojima and Jurka 2011). Thus, in the absence of additional evidence from these fungal species, we consider these as Enterprise-like elements. Regardless, these results suggest that Enterprise is a YR-mobilized group of DNA transposons that is spread throughout fungal genomes.

\section{The Spok block can be deleterious}

In $P$. anserina, wild strains with more than one full copy of the Spok block have never been found, although it is easy to generate them in the lab via crosses (Vogan, Ament-Velásquez, et al. 2019). It is possible that the burden imposed by the Spok block on the host is quite high, leading to selection to purge most copies in nature. To evaluate this hypothesis, we made use of backcrosses of two of the Psk strains (Vogan, Ament-Velásquez, et al. 2019). The backcrossed strains Psk1xS ${ }_{14}$ and Psk7xS ${ }_{14}$ are isogenic with the reference strain S, except that $S$ has no Spok block. Psk1xS 14 has the Spok block on chromosome 3 and induces killing in $90 \%$ of meioses, and Psk7xS 14 has the Spok block on the left arm of chromosome 5 and induces spore killing in $50 \%$ of meioses. We crossed strains either to themselves (no spore killing) or to strain S (spore killing) and evaluated a number of traits related to fitness. Radial growth and time to germination of spores produced by the matings showed no variation between crosses (data not shown), however significant differences were observed among the amount of spores produced by a cross (Figure 3). Specifically, crosses from selfings of Psk1xS ${ }_{14}$ produced significantly fewer spores than from selfings of either $\mathrm{S}$ or Psk7xS $\mathrm{S}_{14}$, despite the fact that no spore killing occurs, indicating that the Psk-1 Spok block inhibits spore production. This effect was even greater in crosses between $\mathrm{S}$ and $\mathrm{Psk}_{1 \times S_{14}}$, and was most prominent when Psk1xS $\mathrm{S}_{14}$ was used as the female, suggesting a maternal effect. With both Psk1xS 14 and Psk7xS P $_{14}$ more spores were produced in the killing crosses than expected given the proportion of killing per ascus (Vogan, Ament-Velásquez, et al. 2019). As there were no significant differences in the 
amount of spores produced by Psk7xS 14 than by $\mathrm{S}$, this result suggests that the general presence of the Spok block itself is not deleterious, but rather that the negative effect is due to the specific content and/or to the genomic location of a given Spok block.

\section{Discussion}

Here we provide evidence that the Spok block is a variant of a newly described TE, Enterprise, that moves throughout the genome of $P$. anserina by means of YR-mediated transposition. In addition to this ability to move, the Spok block is also capable of meiotic drive due to the presence of the Spok genes. Given the union of these two selfish properties, it can be asked who is parasitizing whom. One possibility is that Enterprise has hijacked the Spoks in order to increase its rate of transmission, thereby parasitizing a resident genomic parasite and becoming a genomic hyperparasite. Support for this commandeering can be found in the distribution of the Spok genes. In both $P$. anserina and $P$. comata, the Spok genes that are not found in association with the Spok block are at high frequency (Spok2 is found in $98 \%$ of strains isolated in Wageningen (Vogan, Ament-Velásquez, et al. 2019), and so far Spok1 has been found in all analysed strains of $P$. comata). The Spok block is comparatively rare ( $18 \%$ of strains from Wageningen (Vogan, Ament-Velásquez, et al. 2019)), indicating that it may prevent the Spoks from reaching high frequencies. Alternatively, the Spok genes may generally benefit from moving throughout the genome. Spok1 and Spok2 are found at different locations in the genome and are surrounded by TEs, suggesting they may have moved through other mechanisms like TE-mediated ectopic recombination (Vogan, Ament-Velásquez, et al. 2019). It may thus be advantageous for the Spoks to mobilize within TEs, like Enterprise, in order to change their genomic position on a regular basis, due to the fact that this relocation will result in a novel spore killing phenotype. Presumably, the population dynamics of meiotic drive ultimately decide the fate of the Spoks (Nauta and Hoekstra 1993, Martinossi-Allibert, et al. 2020), but the confederation of the Spoks and Enterprise as the Spok block may fundamentally change how effective selection is at controlling either element.

Given that the results of the fitness experiments suggest that the Spok block can be deleterious, there may be strong selection to purge any copies of Enterprise, but this may ultimately be dependent on genomic context as insinuated by the differences in phenotypic effect of carrying the Psk-1 and Psk-7 Spok blocks. The Psk-7 Spok block is larger by nearly 50 $\mathrm{kb}$, some of which includes known retrotransposons. Yet, only Psk-1 showed a significant decrease in the amount of spores produced. It thus seems probable that the location of the block has a stronger deleterious effect rather than its content or size. The Psk-1 Spok block is located close to the centromere on the left arm of chromosome 3. It is possible that the increased amount of killing in Psk-1 results in its poorer performance, although this cannot explain the observed maternal effect. The rDNA cluster resides on the same chromosome arm as the Psk-1 block (Espagne et al. 2008). Given that this arm is only $\sim 700 \mathrm{~kb}$ and the block itself is $113 \mathrm{~kb}$, the Spok block insertion might interfere with recombination, which is necessary for proper segregation of rDNA (Tomson et al. 2006) and likely inhibits the ability of the strain to produce viable spores. Moreover, the observation that there is not a large decrease in spore production for Psk-1 or Pks-7 strains when involved in spore killing suggests that strains are 
able to compensate for the lost spores in some way. This latter result has significant implications to our understanding of spore killing as meiotic drive, as it shifts the system away from providing the killer genotype a relative fitness advantage to an absolute fitness advantage (Nauta and Hoekstra 1993; Lyttle 1991; Martinossi-Allibert et al. 2020). Explicitly, in the naïve expectation, a spore killer that is $100 \%$ efficient would reduce the total amount of spores produced in a killing cross to half. Thus, it does not produce more total offspring with its genotype than a non-spore killing gene (relative advantage). However, if the strain carrying the spore killer is able to compensate for the loss of spore production, and produce the same or similar number of spores as in a non-killing cross (as observed here), the spore killer observes an absolute advantage, as is the case for other types of meiotic drive, like female meiotic drive. Therefore, spore killers may be more successful at invading and driving through populations than previously thought.

The Enterprise clearly has the ability to move large amounts of genetic material around the genome and TEs are known to be agents of horizontal gene transfer (HGT) (Schaack, Gilbert, and Feschotte 2010; Gilbert and Feschotte 2018). As such, it is plausible that Enterprise and related YR-mobilized DNA transposons may jump between species, and thereby transport any additional genes they may carry. The phylogeny of the Kirc homologs is indicative of HGT as it shows closely related Kirc homologs distributed amongst unrelated fungi. In at least two cases, fungal TEs have been implicated as the vehicles for gene mobility of adaptive genes. In the first case, a hAT element is associated with the HGT of toxin genes among cereal pathogens (McDonald et al. 2019). The second case comes from a recent publication which described a TE named HEPHAESTUS in the fungus Paecilomyces (Urquhart et al. 2020). It carries multiple genes that provide resistance to at least five different heavy metals and shows evidence of transfer with a distantly related species of Penicillium. In the latter example the TE responsible for enacting the transposition is unidentified, but strikingly, a gene with a DUF3435 domain is present at the start of HEPHAESTUS (annotated as $h h p A$ ). We find little similarity between Kirc and $h h p A$ at the protein level, but our analyses here strongly suggest that $h h p A$ is also a YR and may be responsible for the reported transposition. Whether the Enterprise itself can also play a role in adaptive HGT is unknown as of yet, but the potential certainly exists. We note that most of the fungal genomes in which HGT has been reported (Wisecaver and Rokas 2015) possess genes containing DUF3435 domains, which together with the data from HEPHAESTUS suggests a convergent role in this type of TE as a vector for HGT of adaptive genes in fungi.

\section{Conclusions}

363 The constant 'tug-of-war' between TEs linking themselves to host genes, and the actions of genome defense and selection to purge them is of key importance to the evolution of genome architecture. It is likely that we are witnessing this fight play out to the extreme in $P$. anserina, with the high effectiveness of both RIP and the Spok block, making Podospora an ideal system in which to continue to study genomic conflict. The discovery that the up to $247 \mathrm{~kb}$ large Spok block likely transposes through a YR-mediated mechanism moves the upper limit of TE size by nearly $100 \mathrm{~kb}$, and its hyper-selfishness combining TE mobility and meiotic drive adds a new 
dynamic by which selfish elements exploit their host genome. This study not only changes our perception of how TEs influence genome evolution, but also broadens the horizons in terms of what may be possible through genetic manipulations in the laboratory. Understanding the molecular mechanism of YR-mediated transposition should thus be of prime interest in future research.

\section{Methods}

Strains used in this study were obtained from the Wageningen collection and cultivated as in Vogan, Ament-Velásquez, et al. (2019). Strain S was used as the standard reference strain with no Spok block. As spore killer strains for the fitness experiments (see below) we used the backcrossed strains Psk1xS 14 and Psk7xS 14 (Vogan, Ament-Velásquez, et al. 2019), which should be isogenic to $S$ but with a Spok block in chromosome 3 and 5, respectively. The more recently isolated strains Wa131, Wa137 and Wa139 were sampled during the fall of 2016 around Wageningen (the Netherlands) from dung of rabbit (Wa131 and Wa137, locality Unksepad Oosterbeek) or horse (Wa139, locality Uiterwaarden Wolfswaard). Morphological differences like smaller perithecia and abundant tomentose apricot-colored mycelium in HPM medium (Vogan, Ament-Velásquez, et al. 2019), as well as analyses of sequence data, allowed us to assign Wa131 and Wa139 to the species $P$. comata. Previously, only one strain from this species, $T_{D}$, was known (Boucher, Nguyen, and Silar 2017; Vogan, Ament-Velásquez, et al. 2019), hence these new strains constitute a new report of this species for the Netherlands.

\section{DNA and RNA extraction and sequencing}

Following Vogan et al. (Vogan, Ament-Velásquez, et al. 2019), we grew monokaryotic strains on PASM0.2 plates covered with a layer of cellophane. Genomic DNA for short-read sequencing was extracted from $80-100 \mathrm{mg}$ of fungal tissue with the Fungal/Bacterial Microprep kit (Zymo; www.zymo.com). Paired-end libraries were prepared and sequenced using the Illumina HiSeq $X$ (150-bp-long) technology at the SNP and SEQ Technology platform (SciLifeLab, Uppsala, Sweden). For RNA extraction, around $150 \mathrm{mg}$ of harvested mycelium were frozen in liquid nitrogen and stored at $-80^{\circ} \mathrm{C}$. We extracted total RNA from the grounded frozen tissue using the RNeasy Plant Mini Kit (Qiagen, Hilden, Germany). Quality was checked on the agilent 2100 Bioanalyzer (Agilent Technologies, USA) and the RNA was treated with DNasel (Thermo 
407

408

409

410

Poly(A) mRNA Magnetic Isolation Module (New England Biolabs). A paired-end library was sequenced with Illumina HiSeq 2500 at the SNP and SEQ Technology platform.

For long-read sequencing, we grew the monokaryotic strains in PASM0.2 plates, from where we sliced small agar cubes to inoculate liquid cultures of $200 \mathrm{ml} 3 \%$ malt extract solution, which were subsequently incubated in a shaker for $10-14$ days at $27^{\circ} \mathrm{C}$ (Vogan, AmentVelásquez, et al. 2019). Mycelium aggregates were filtered from the flasks, any remaining agar was removed, and around $1 \mathrm{~g}$ was stored at $-20^{\circ} \mathrm{C}$. As described in Sun et al. (2017), the tissue was freeze-dried and macerated, followed by DNA extraction using Genomic Tip G-500 columns (Qiagen) and cleaning with the PowerClean DNA Clean-Up kit (MoBio Labs). Additionally, DNA was purified using magnetic beads (Speed-Beads, GE) and eluted for $20 \mathrm{~min}$ at $37^{\circ} \mathrm{C}$ followed by overnight storage at $4^{\circ} \mathrm{C}$ twice to increase concentration (around $65 \mathrm{ng} / \mathrm{\mu l}$ ). Wa137- was sequenced on an R9.5.1 Flowcell (Oxford Nanopore Technologies) with a modified SQK-RAD004 protocol using $550 \mathrm{ng}$ DNA to $1.5 \mu \mathrm{FRA}$ to increase read lengths. Wa139- was prepared using the ligation protocol (SQK-LSK109) and sequenced on an R9.4.1 flowcell. Basecalling was done using Guppy v. 1.6.

\section{Genome assembly}

For most strains we used the assemblies produced in Vogan \& Ament-Velásquez et al. (2019). For newly sequenced strains, we produced new assemblies as follows. The adapters from the Illumina HiSeq reads were identified with cutadapt v. 1.13 (Martin 2011) and removed using Trimmomatic v. 0.36 (Bolger, Lohse, and Usadel 2014) using the following options: ILLUMINACLIP:adapters.fasta:1:30:9 LEADING:20 TRAILING:20 SLIDINGWINDOW:4:20 MINLEN:30. Pairs with both forward and reverse reads after filtering were used for downstream analyses. For the strain Wa131, which only has Illumina data, we used SPAdes v. 3.12.0 (Bankevich et al. 2012) with the k-mers 21,33,55,77 and the --careful option. For the strains Wa137 and Wa139, the MinION reads with a mean Phred quality (QV) above 9 and longer than $1 \mathrm{~kb}$ were assembled using Minimap2 v. 2.11 and Miniasm v. 0.2 (Li 2018, 2016). The resulting assembly was polished twice with Racon v. 1.3.1 (Vaser et al. 2017) using all MinlON reads (no filtering). Further polishing was done with the filtered Illumina reads in five consecutive rounds of Pilon v. 1.22 (Walker et al. 2014). We used BWA v. 0.7.17 (Li and Durbin 2010) for short-read mapping, with PCR duplicates marked using Picard v. 2.18.11 (http://broadinstitute.github.io/picard/), as well as local indel re-alignment using the Genome Analysis Toolkit (GATK) v. 3.7 (Van der Auwera et al. 2013).

We assigned the scaffolds to chromosomes based on alignments to the reference genome of the S strain (Espagne et al. 2008), available at the Joint Genome Institute MycoCosm website (https://mycocosm.jgi.doe.gov/mycocosm/home) as "Podan2" (Grigoriev et al. 2014). We discarded small contigs ( $<100 \mathrm{~kb})$ of rDNA repeats as well as mitochondrialderived sequences, except for the largest mitochondrial contig. We assessed the quality of the final assemblies by visual inspection of the mapping of both long and short reads using Minimap2 and BWA, respectively. Mean depth of coverage was calculated with QualiMap v.2.2 
(Okonechnikov, Conesa, and García-Alcalde 2016). Other assembly statistics were calculated with QUAST v. 4.6 .3 (Mikheenko et al. 2016).

\section{Genome annotation}

A GitHub repository is available with Snakemake v. 5.4 .4 (Köster and Rahmann 2012) pipelines relevant to genome annotation at https://github.com/johannessonlab/SpokBlockPaper.

The TEs and other repeats in $P$. anserina were classified previously by Espagne et al. (2008) based on the original reference genome of the S strain or "Podan1", and is hereafter referred to as the "Espagne library". To explore the diversity of TEs in the newly generated Podospora genomes, we identified repeats de novo and manually compared them to the Espagne library to identify duplicates and new elements. Specifically, we ran RepeatModeler v. 1.0.8 (http://www.repeatmasker.org/RepeatModeler/) on the scaffolds larger than $50 \mathrm{~kb}$ of all available long-read assemblies (Snakemake pipeline PaTEs.smk). Each resulting RepeatModeler consensus was BLASTn-searched back to the original genome and the best 20 hits with 2-kb flanks were aligned with T-Coffee v. 12.00.7fb08c2 (Notredame, Higgins, and Heringa 2000) (TEManualCuration.smk), and visually inspected for manual curation. The curated consensuses were assigned to the Espagne et al. (2008) equivalents based on similarity (allowing for RIP-induced mutations) or were given a new name when having no homology to anything in the Espagne library. It was discovered that the gypsy element crapaud has numerous diverged copies with unique LTRs. We annotated all crapaud LTRs that were in multiple copies within $P$. anserina individually to improve repeat masking. We refer to the final repeat library as "PodoTE-1.00" (available at the GitHub repository).

To generate a genome annotation of all assemblies, we ran an updated version of the pipeline in Vogan \& Ament-Velásquez et al. (2019), named PaAnnotation.smk. We used MAKER v. 3.01.2 (Holt and Yandell 2011; Campbell et al. 2014) with the previously produced training files used for the ab initio prediction programs GeneMark-ES v. 4.32 (Lomsadze et al. 2005; Ter-Hovhannisyan et al. 2008) and SNAP release 2013-06-16 (Lomsadze et al. 2005), as well as the following dependencies: RepeatMasker v. 4.0 .7 (http://www.repeatmasker.org/), BLAST suite 2.6.0+ (Camacho et al. 2009), Exonerate v. 2.2.0 (Slater and Birney 2005), and tRNAscan-SE v. 1.3.1 (Lowe and Eddy 1997). As evidence, we used STAR v. 2.6.1b (Dobin et al. 2013) to produce transcript models (maximum intron length set to $1000 \mathrm{bp}$ ) of various RNAseq data sets. Specifically, we mapped the reads of the monokaryotic isolate Wa63- $(P$. anserina) to the assembly PaWa63m (Vogan, Ament-Velásquez, et al. 2019), of the monokaryotic isolate Wa131- ( $P$. comata) to the assembly PcWa139m (this study), and of the dikaryotic Psk7xS ${ }_{14}(P$. anserina) to the assembly PaWa58m (Vogan, Ament-Velásquez, et al. 2019). We then processed the mapped reads with Cufflinks v. 2.2 .1 (Trapnell et al. 2010) to obtain the transcript models. As external evidence, we used CDS from the Podan2 annotation, protein sequences from the T strain of $P$. comata (Silar et al. 2019), and a small dataset of manually curated proteins. To aid in manual curation of selected regions (mostly the Spok 
as the output of RepeatMasker ran externally from MAKER with the PodoTE-1.00 library. Additionally, we queried predicted gene models into the NCBI databases (NCBI Resource Coordinators 2016) to verify the annotations.

The Kirc protein sequence was analyzed with HHPred (Zimmermann et al. 2018) and Gremlin (Balakrishnan et al. 2011). The Gremlin-generated alignment of Kirc homologs was used to generate region-specific sequence logos with Weblogo (Crooks et al. 2004). The relationship of Kirc to other YRs was confirmed by comparing the sequence to the crystal structure of known YRs (CRE (PDB code 3mgv), XERD (1a0p), and FLP (1flo)) as well as the protein sequence from the transposable element Crypton-Cn1 using the software Promals3D (Pei, Kim, and Grishin 2008).

\section{Genome alignments}

502 We used the NUCmer program from the MUMmer package v. 4.0.0beta2 (Kurtz et al. 2004) using the parameters - $b 200$-c 22 --maxmatch to align the Spok blocks to each other, and changed to -c 40 for whole-genome assemblies. To achieve higher sensitivity, we used BLASTn from the BLAST suite 2.9.0 (Camacho et al. 2009) to search for the presence of the unclassified repeats bufo and schoutenella. Both the NUCmer and the BLAST alignments were plotted using Circos v. 0.69.6 (Krzywinski et al. 2009) along with manual curations of coding regions and repetitive elements. The distribution of TE and gene content along chromosomes was calculated in windows of $50 \mathrm{~kb}$ with steps of $10 \mathrm{~kb}$ using BEDtools v. 2.29.0 (Quinlan and Hall 2010; Quinlan 2014), with the utilities makewindows and coverage. The fraction of conservation between blocks compared to the block in Wa137 was calculated by aligning the block sequences (within the TSD) of Wa28 (Psk-2), Wa53 (Psk-1), Wa58 (Psk-7) and Wa139 with NUCmer and the BEDtools utility genomecov. The Snakemake pipelines used to produce the Circos plots (CircosBlock.smk and CircosAllBlocks.smk) are available at https://github.com/johannessonlab/SpokBlockPaper.

\section{Phylogenetic analyses}

Homologs of Kirc were identified from GenBank using BLASTp with the truncated version of Kirc from Wa53 that has no CHROMO domain. Nucleotide sequences of hits with e-values $<1 \mathrm{e}-100$ were compiled along with a homolog from $P$. anserina (Pa_5_10116), and two homologs from Melanconium sp. NRRL 54901 extracted from MycoCosm (see below), and aligned with MACSE v. 2.03 (Ranwez et al. 2018). We used TrimAl v. 1.4.1 (Capella-Gutierrez, SillaMartinez, and Gabaldon 2009) to trim the resulting protein alignment with the -gappyout option. We then used IQTree v. 1.6.8 (Kalyaanamoorthy et al. 2017; Nguyen et al. 2014) to produce a Maximum Likelihood phylogeny with extended model selection (-m MFP). To estimate the branch support, we produced 1000 standard bootstrap pseudoreplicates.

To search Mycocosm for other copies of Enterprise the following approach was taken. 
530 identified and the regions with putative Kirc homologs were manually extracted. Priority was given to genomes where the hits were associated with large duplicated regions (>50 kb). Melanconium sp. NRRL 54901 (produced as part of the 1KFG project; Spatafora et al. 2011) had the most copies with clear termini.

\section{Fitness assays}

The cultures used for the crosses were revived from the -80 freezer on PASM0.2 (van Diepeningen et al. 2008) at $27^{\circ} \mathrm{C}$ for several days and then stored at $+4^{\circ} \mathrm{C}$ until use. Strains were grown for 5 days on fresh PASM0.2 plates before inoculating the cross. In the crosses, one strain was grown as mycelia and thereby assigned the female role, while a compatible strain of the other mating type was assigned the male role by fertilizing the mycelia with microconidia. The strain that was assigned the female role was grown in a $35 \mathrm{~mm}$ petri dish with $5 \mathrm{ml}$ HPM medium (Vogan, Ament-Velásquez, et al. 2019) by inoculating a small cube of agar with mycelium ( $2 \times 2 \mathrm{~mm}$ ). In parallel, the strain that was assigned the male role was grown on a $90 \mathrm{~mm}$ petri dish with micro conidiation medium (Esser 1974; King 2013) by inoculating seven plugs of mycelium spread over the plate. After 7 days of growth, microconidia were harvested by adding $5 \mathrm{ml}$ of sterile water to the plate and sweep over the mycelium with a drigalski spatula for 1 minute. The female mycelium was then fertilized with $0.5 \mathrm{ml}$ of the microconidial suspension. The suspension was carefully spread out to make sure all mycelium was covered. The fertilized mycelia were then further incubated under standard conditions $\left(27^{\circ} \mathrm{C}, 12 / 12\right.$ light/dark cycle) (Vogan, Ament-Velásquez, et al. 2019). The cultures were monitored daily for signs of spores shot from the asci in order to score the first day of spore-shooting. To reduce the complexity of the experiment, the strains used as female were always of mating type mat+.

At 6 days post-fertilization, single spores were collected with a needle to measure germination frequency and growth speed. From each cross, 10 spores from 4-spored asci were picked, and in cases with spore killing, an additional 10 spores from 2-spored asci were picked. The 10 spores were transferred to a single $90 \mathrm{~mm}$ petri dish with PASM2 medium (van Diepeningen et al. 2008) with $0.4 \%$ ammonium acetate added (to activate the spores) (Esser 1974; King 2013). Spores were spaced out in a predetermined pattern (4 lines of 2, 3, 3, 2 spores). After two days of incubation, the germination was scored and colony diameter was measured in two directions. If there was no growth microscopic inspection was performed to check whether a spore was present in the agar to avoid scoring no germination in case the inoculation failed.

At 12 days post-fertilization, spores were harvested from the lids of each crossed culture and used for estimating total spore yield. Spores were collected by pipetting $750 \mu$ l of harvest liquid (1M NAOH, $0.025 \%$ SDS) in the lid. Spores were then scraped off the lid using the pipette tip. The liquid was then collected into a $2 \mathrm{ml}$ Eppendorf tube. Another $750 \mu \mathrm{l}$ of harvest liquid was used to repeat the process to make sure most of the spores were collected from the lid. The tubes were then heated for 4 hours at $85^{\circ} \mathrm{C}$, then shaken in a Qiagen Tissuelyser for 90 seconds at $30 \mathrm{~Hz}$. After this, the tubes were stored at $4^{\circ} \mathrm{C}$ overnight. The cooled tubes were again shaken in a Qiagen Tissuelyser for 90 seconds at $30 \mathrm{~Hz}$. This process prevents the clumping of spores. Total yield was determined by counting the amount of spores in a volume of 
$5715 \mu \mathrm{l}$ of $50 \mathrm{x}$ diluted suspension pipetted on an object glass using a stereomicroscope. Counts 572 were taken five times for each replicate cross. Statistical analyses were conducted in base R v.

$573 \quad 3.5 .0$ to determine significance and power.

\section{Acknowledgements}

575 This work was supported by the European Research Council (ERC) grant ERC-2014-CoG 576 (project 648143, SpoKiGen) and The Swedish Research Council to H.J, and support from the 577 Lars Hierta Memorial Foundation and The Nilsson-Ehle Endowments of the Royal 578 Physiographic Society of Lund to S.L.A.V. We thank the support given by the National 579 Genomics Infrastructure (NGI) / Uppsala Genome center on massive parallel DNA sequencing. 580 The computations were performed on resources provided by SNIC through Uppsala 581 Multidisciplinary Center for Advanced Computational Science (UPPMAX) under the projects 582 SNIC 2017/1-567 and SNIC 2019/8-371. The sequence data of Melanconium sp. NRRL 54901 583 was produced by the US Department of Energy Joint Genome Institute http://www.jgi.doe.gov/ 584 in collaboration with the user community. We would also like to thank Sergio Tusso and the TE 585 Jamboree of the Suh's Lab for useful advice.

References

Arkhipova, Irina R., and Irina A. Yushenova. 2019. "Giant Transposons in Eukaryotes: Is Bigger Better?" Genome Biology and Evolution 11 (3): 906-18.

Balakrishnan S, Kamisetty H, Carbonell JG, Lee SI, Langmead CJ. 2011. Learning generative models for protein fold families. Proteins: Structure, Function, and Bioinformatics 79: 10611078.

Bankevich, Anton, Sergey Nurk, Dmitry Antipov, Alexey A. Gurevich, Mikhail Dvorkin, Alexander S. Kulikov, Valery M. Lesin, et al. 2012. "SPAdes: A New Genome Assembly Algorithm and Its Applications to Single-Cell Sequencing." Journal of Computational Biology: A Journal of Computational Molecular Cell Biology 19 (5): 455-77.

Bennett, E. A., H. Keller, R. E. Mills, S. Schmidt, J. V. Moran, O. Weichenrieder, and S. E. Devine. 2008. "Active Alu Retrotransposons in the Human Genome." Genome Research 18 (12): 1875-1883.

Bolger, Anthony M., Marc Lohse, and Bjoern Usadel. 2014. "Trimmomatic: A Flexible Trimmer for Illumina Sequence Data." Bioinformatics 30 (15): 2114-20.

Boucher, Charlie, Tinh-Suong Nguyen, and Philippe Silar. 2017. "Species Delimitation in the Podospora anserina/P. pauciseta/P. comata Species Complex (Sordariales)." Cryptogamie, Mycologie. 38 (4): 485-506.

Camacho, Christiam, George Coulouris, Vahram Avagyan, Ning Ma, Jason Papadopoulos, Kevin Bealer, and Thomas L. Madden. 2009. "BLAST+: Architecture and Applications." BMC Bioinformatics 10: 421.

Cambareri, E., B. Jensen, E. Schabtach, and E. Selker. 1989. "Repeat-Induced G-C to A-T Mutations in Neurospora." Science. 244 (4912): 1571-1575.

Campbell, Michael S., Carson Holt, Barry Moore, and Mark Yandell. 2014. "Genome Annotation and Curation Using MAKER and MAKER-P." Current Protocols in Bioinformatics 48: 
613

614

615

616

617

618

619

620

621

622

623

624

625

626

627

628

629

630

631

632

633

634

635

636

637

638

639

640

641

642

643

644

645

646

647

648

649

650

651

652

653

654

655

656

657

658

659

660

661

662

663
4.11.1-39.

Capella-Gutierrez, S., J. M. Silla-Martinez, and T. Gabaldon. 2009. "trimAl: A Tool for Automated Alignment Trimming in Large-Scale Phylogenetic Analyses." Bioinformatics 25 (15): 1972-1973.

Chénais, Benoît, Aurore Caruso, Sophie Hiard, and Nathalie Casse. 2012. "The Impact of Transposable Elements on Eukaryotic Genomes: From Genome Size Increase to Genetic Adaptation to Stressful Environments." Gene 509 (1): 7-15.

Crooks GE, Hon G, Chandonia JM, Brenner SE. 2004. WebLogo: a sequence logo generator. Genome Research 14: 1188-1190.

Diepeningen, Anne D. van, Alfons J. M. Debets, S. Marijke Slakhorst, and Rolf F. Hoekstra. 2008. "Mitochondrial pAL2-1 Plasmid Homologs Are Senescence Factors in Podospora anserina Independent of Intrinsic Senescence." Biotechnology Journal 3 (6): 791-802.

Dobin, Alexander, Carrie A. Davis, Felix Schlesinger, Jorg Drenkow, Chris Zaleski, Sonali Jha, Philippe Batut, Mark Chaisson, and Thomas R. Gingeras. 2013. "STAR: Ultrafast Universal RNA-Seq Aligner." Bioinformatics 29 (1): 15-21.

El-Gebali, Sara, Jaina Mistry, Alex Bateman, Sean R. Eddy, Aurélien Luciani, Simon C. Potter, Matloob Qureshi, et al. 2019. "The Pfam Protein Families Database in 2019." Nucleic Acids Research 47 (D1): D427-32.

Espagne, Eric, Olivier Lespinet, Fabienne Malagnac, Corinne Da Silva, Olivier Jaillon, Betina M. Porcel, Arnaud Couloux, et al. 2008. "The Genome Sequence of the Model Ascomycete Fungus Podospora anserina." Genome Biology 9 (5): R77.

Esposito, D., and J. J. Scocca. 1997. "The Integrase Family of Tyrosine Recombinases: Evolution of a Conserved Active Site Domain." Nucleic Acids Research 25 (18): 3605-14.

Gaag, M. van der, A. J. Debets, J. Oosterhof, M. Slakhorst, J. A. Thijssen, and R. F. Hoekstra. 2000. "Spore-Killing Meiotic Drive Factors in a Natural Population of the Fungus Podospora anserina." Genetics 156 (2): 593-605.

Gilbert, Clément, and Cédric Feschotte. 2018. "Horizontal Acquisition of Transposable Elements and Viral Sequences: Patterns and Consequences." Current Opinion in Genetics \& Development 49: 15-24.

Grigoriev IV, Nikitin R, Haridas S, Kuo A, Ohm R, Otillar R, Riley R, Salamov A, Zhao X, Korzeniewski F, Smirnova T, Nordberg H, Dubchak I, Shabalov I. (2014) "MycoCosm portal: gearing up for 1000 fungal genomes." Nucleic Acids Research 42(1): D699-704.

Grognet, Pierre, Hervé Lalucque, Fabienne Malagnac, and Philippe Silar. 2014. "Genes That Bias Mendelian Segregation." PLoS Genetics 10 (5): e1004387.

Haas, Brian J., Sophien Kamoun, Michael C. Zody, Rays H. Y. Jiang, Robert E. Handsaker, Liliana M. Cano, Manfred Grabherr, et al. 2009. "Genome Sequence and Analysis of the Irish Potato Famine Pathogen Phytophthora infestans." Nature 461 (7262): 393-98.

Haas, Brian J., Alexie Papanicolaou, Moran Yassour, Manfred Grabherr, Philip D. Blood, Joshua Bowden, Matthew Brian Couger, et al. 2013. "De Novo Transcript Sequence Reconstruction from RNA-Seq Using the Trinity Platform for Reference Generation and Analysis." Nature Protocols 8 (8): 1494-1512.

Holt, Carson, and Mark Yandell. 2011. "MAKER2: An Annotation Pipeline and GenomeDatabase Management Tool for Second-Generation Genome Projects." BMC Bioinformatics $12: 491$.

Inoue, Yusuke, Masahiko Kumagai, Xianbo Zhang, Tomonori Saga, Deshou Wang, Akihiko Koga, and Hiroyuki Takeda. 2018. "Fusion of piggyBac-like Transposons and Herpesviruses Occurs Frequently in Teleosts." Zoological Letters 4 (1): 6.

Inoue, Yusuke, Tomonori Saga, Takumi Aikawa, Masahiko Kumagai, Atsuko Shimada, Yasushi Kawaguchi, Kiyoshi Naruse, Shinichi Morishita, Akihiko Koga, and Hiroyuki Takeda. 2017. "Complete Fusion of a Transposon and Herpesvirus Created the Teratorn Mobile Element in Medaka Fish." Nature Communications 8 (1): 551. 
664

665

666

667

668

669

670

671

672

673

674

675

676

677

678

679

680

681

682

683

684

685

686

687

688

689

690

691

692

693

694

695

696

697

698

699

700

701

702

703

704

705

706

707

708

709

710

711

712

713

714
Jurka, Jerzy. 2004. "Evolutionary Impact of Human Alu Repetitive Elements." Current Opinion in Genetics \& Development 14 (6): 603-8.

Kalyaanamoorthy, Subha, Bui Quang Minh, Thomas K. F. Wong, Arndt von Haeseler, and Lars S. Jermiin. 2017. "ModelFinder: Fast Model Selection for Accurate Phylogenetic Estimates." Nature Methods 14 (6): 587-89.

King, Robert C. 2013. Handbook of Genetics: Volume 1 Bacteria, Bacteriophages, and Fungi. Springer Science \& Business Media.

Kojima, Kenji K., and Jerzy Jurka. 2011. "Crypton Transposons: Identification of New Diverse Families and Ancient Domestication Events." Mobile DNA 2 (1): 12.

Kordis, Dusan. 2005. "A Genomic Perspective on the Chromodomain-Containing Retrotransposons: Chromoviruses." Gene 347 (2): 161-73.

Köster, Johannes, and Sven Rahmann. 2012. "Snakemake - a Scalable Bioinformatics Workflow Engine." Bioinformatics 28 (19): 2520-22.

Krzywinski, Martin, Jacqueline Schein, Inanç Birol, Joseph Connors, Randy Gascoyne, Doug Horsman, Steven J. Jones, and Marco A. Marra. 2009. "Circos: An Information Aesthetic for Comparative Genomics." Genome Research 19 (9): 1639-45.

Kurtz, Stefan, Adam Phillippy, Arthur L. Delcher, Michael Smoot, Martin Shumway, Corina Antonescu, and Steven L. Salzberg. 2004. "Versatile and Open Software for Comparing Large Genomes." Genome Biology 5 (2): R12.

$\mathrm{Li}$, Heng. 2016. "Minimap and Miniasm: Fast Mapping and de Novo Assembly for Noisy Long Sequences." Bioinformatics 32 (14): 2103-10.

_. 2018. "Minimap2: Pairwise Alignment for Nucleotide Sequences." Bioinformatics 34 (18): 3094-3100.

$\mathrm{Li}$, Heng, and Richard Durbin. 2010. "Fast and Accurate Long-Read Alignment with BurrowsWheeler Transform." Bioinformatics 26 (5): 589-95.

Linheiro, Raquel S., and Casey M. Bergman. 2012. "Whole Genome Resequencing Reveals Natural Target Site Preferences of Transposable Elements in Drosophila melanogaster." PloS One 7 (2): e30008.

Lomsadze, Alexandre, Vardges Ter-Hovhannisyan, Yury O. Chernoff, and Mark Borodovsky. 2005. "Gene Identification in Novel Eukaryotic Genomes by Self-Training Algorithm." Nucleic Acids Research 33 (20): 6494-6506.

Lowe, T. M., and S. R. Eddy. 1997. "tRNAscan-SE: A Program for Improved Detection of Transfer RNA Genes in Genomic Sequence." Nucleic Acids Research 25 (5): 955-64.

Lyttle, T. W. 1991. "Segregation Distorters." Annual Review of Genetics 25: 511-57.

Martin, Marcel. 2011. "Cutadapt Removes Adapter Sequences from High-Throughput Sequencing Reads." EMBnet.journal. 17 (1): 10-12.

Ivain Martinossi-Allibert, Carl Veller, S. Lorena Ament-Velásquez, Aaron A. Vogan, Claus Rueffler, Hanna Johannesson. 2020. "Invasion and maintenance of spore killing in ascomycete fungi." bioRxiv 2020.04.06.026989.

McDonald, Megan C., Adam P. Taranto, Erin Hill, Benjamin Schwessinger, Zhaohui Liu, Steven Simpfendorfer, Andrew Milgate, and Peter S. Solomon. 2019. "Transposon-Mediated Horizontal Transfer of the Host-Specific Virulence Protein ToxA between Three Fungal Wheat Pathogens." mBio 10 (5): e01515-19.

Mieczkowski, Piotr A., Francene J. Lemoine, and Thomas D. Petes. 2006. "Recombination between Retrotransposons as a Source of Chromosome Rearrangements in the Yeast Saccharomyces cerevisiae." DNA Repair 5 (9-10): 1010-20.

Mikheenko, Alla, Gleb Valin, Andrey Prjibelski, Vladislav Saveliev, and Alexey Gurevich. 2016. "Icarus: Visualizer for de Novo Assembly Evaluation." Bioinformatics 32 (21): 3321-23.

Möller, Mareike, and Eva H. Stukenbrock. 2017. "Evolution and Genome Architecture in Fungal Plant Pathogens." Nature Reviews. Microbiology 15 (12): 771.

Nauta, M. J., and R. F. Hoekstra. 1993. "Evolutionary Dynamics of Spore Killers." Genetics 135 
715

716

717

718

719

720

721

722

723

724

725

726

727

728

729

730

731

732

733

734

735

736

737

738

739

740

741

742

743

744

745

746

747

748

749

750

751

752

753

754

755

756

757

758

759

760

761

762

763

764

765
(3): 923-30.

NCBI Resource Coordinators. 2016. "Database Resources of the National Center for Biotechnology Information." Nucleic Acids Research 44 (D1): D7-19.

Nguyen, Lam-Tung, Heiko A. Schmidt, Arndt von Haeseler, and Bui Quang Minh. 2014. "IQTREE: A Fast and Effective Stochastic Algorithm for Estimating Maximum-Likelihood Phylogenies." Molecular Biology and Evolution 32 (1): 268-74.

Notredame, C., D. G. Higgins, and J. Heringa. 2000. "T-Coffee: A Novel Method for Fast and Accurate Multiple Sequence Alignment." Journal of Molecular Biology 302 (1): 205-17.

Okonechnikov, Konstantin, Ana Conesa, and Fernando García-Alcalde. 2016. "Qualimap 2: Advanced Multi-Sample Quality Control for High-Throughput Sequencing Data." Bioinformatics 32 (2): 292-94.

Pei, Jimin, Bong-Hyun Kim, and Nick V. Grishin. 2008. "PROMALS3D: A Tool for Multiple Protein Sequence and Structure Alignments." Nucleic Acids Research 36 (7): 2295-2300.

Quinlan, Aaron R. 2014. "BEDTools: The Swiss-Army Tool for Genome Feature Analysis." Current Protocols in Bioinformatics 47: 11.12.1-34.

Quinlan, Aaron R., and Ira M. Hall. 2010. "BEDTools: A Flexible Suite of Utilities for Comparing Genomic Features." Bioinformatics 26 (6): 841-42.

Ranwez, Vincent, Emmanuel J. P. Douzery, Cédric Cambon, Nathalie Chantret, and Frédéric Delsuc. 2018. "MACSE v2: Toolkit for the Alignment of Coding Sequences Accounting for Frameshifts and Stop Codons." Molecular Biology and Evolution 35 (10): 2582-84.

Schaack, Sarah, Clément Gilbert, and Cédric Feschotte. 2010. "Promiscuous DNA: Horizontal Transfer of Transposable Elements and Why It Matters for Eukaryotic Evolution." Trends in Ecology \& Evolution 25 (9): 537-46.

Schnable, Patrick S., Doreen Ware, Robert S. Fulton, Joshua C. Stein, Fusheng Wei, Shiran Pasternak, Chengzhi Liang, et al. 2009. "The B73 Maize Genome: Complexity, Diversity, and Dynamics." Science 326 (5956): 1112-15.

Selker, E. U., E. B. Cambareri, B. C. Jensen, and K. R. Haack. 1987. "Rearrangement of Duplicated DNA in Specialized Cells of Neurospora." Cell 51 (5): 741-52.

Silar, Philippe, Jean-Marc Dauget, Valérie Gautier, Pierre Grognet, Michelle Chablat, Sylvie Hermann-Le Denmat, Arnaud Couloux, Patrick Wincker, and Robert Debuchy. 2019. "A Gene Graveyard in the Genome of the Fungus Podospora comata." Molecular Genetics and Genomics: MGG 294 (1): 177-90.

Slater, Guy St C., and Ewan Birney. 2005. "Automated Generation of Heuristics for Biological Sequence Comparison." BMC Bioinformatics 6: 31.

Spatafora, Joey. 2011. "1000 fungal genomes to be sequenced." IMA Fungus 2 (2): 41-45.

Sun, Yu, Jesper Svedberg, Markus Hiltunen, Pádraic Corcoran, and Hanna Johannesson. 2017. "Large-Scale Suppression of Recombination Predates Genomic Rearrangements in Neurospora tetrasperma." Nature Communications 8 (1): 1140.

Talla, Venkat, Alexander Suh, Faheema Kalsoom, Vlad Dincă, Roger Vila, Magne Friberg, Christer Wiklund, and Niclas Backström. 2017. "Rapid Increase in Genome Size as a Consequence of Transposable Element Hyperactivity in Wood-White (Leptidea) Butterflies." Genome Biology and Evolution 9 (10): 2491-2505.

Ter-Hovhannisyan, V., A. Lomsadze, Y. O. Chernoff, and M. Borodovsky. 2008. "Gene Prediction in Novel Fungal Genomes Using an Ab Initio Algorithm with Unsupervised Training." Genome Research 18 (12) 1979-1990.

Tomson, Brett N., Damien D'Amours, Brittany S. Adamson, Luis Aragon, and Angelika Amon. 2006. "Ribosomal DNA Transcription-Dependent Processes Interfere with Chromosome Segregation." Molecular and Cellular Biology 26 (16): 6239-47.

Trapnell, Cole, Brian A. Williams, Geo Pertea, Ali Mortazavi, Gordon Kwan, Marijke J. van Baren, Steven L. Salzberg, Barbara J. Wold, and Lior Pachter. 2010. "Transcript Assembly and Quantification by RNA-Seq Reveals Unannotated Transcripts and Isoform Switching 
during Cell Differentiation." Nature Biotechnology 28 (5): 511-15.

Urquhart, A. S., Chong, N. F., Yang, Y., \& Idnurm, A. (2020). "Eukaryotic transposable elements as "cargo carriers": the forging of metal resistance in the fungus Paecilomyces variotii." bioRxiv 2020.03.06.981548.

Van der Auwera, Geraldine A., Mauricio O. Carneiro, Chris Hartl, Ryan Poplin, Guillermo Del Angel, Ami Levy-Moonshine, Tadeusz Jordan, et al. 2013. "From FastQ Data to High Confidence Variant Calls: The Genome Analysis Toolkit Best Practices Pipeline." Current Protocols in Bioinformatics 43: 11.10.1-33.

Vaser, Robert, Ivan Sović, Niranjan Nagarajan, and Mile Šikić. 2017. "Fast and Accurate de Novo Genome Assembly from Long Uncorrected Reads." Genome Research 27 (5): 73746.

Vogan, Aaron A., S. Lorena Ament-Velásquez, Alexandra Granger-Farbos, Jesper Svedberg, Eric Bastiaans, Alfons J. M. Debets, Virginie Coustou, et al. 2019. "Combinations of Spok Genes Create Multiple Meiotic Drivers in Podospora." eLife 8: e46454.

Walker, Bruce J., Thomas Abeel, Terrance Shea, Margaret Priest, Amr Abouelliel, Sharadha Sakthikumar, Christina A. Cuomo, et al. 2014. "Pilon: An Integrated Tool for Comprehensive Microbial Variant Detection and Genome Assembly Improvement." PloS One 9 (11): e112963.

Wicker, Thomas, François Sabot, Aurélie Hua-Van, Jeffrey L. Bennetzen, Pierre Capy, Boulos Chalhoub, Andrew Flavell, et al. 2007. "A Unified Classification System for Eukaryotic Transposable Elements." Nature Reviews. Genetics 8 (12): 973-82.

Wisecaver, Jennifer H., and Antonis Rokas. 2015. "Fungal Metabolic Gene Clusters-Caravans Traveling across Genomes and Environments." Frontiers in Microbiology 6: 161.

Zimmermann L, Stephens A, Nam SZ, Rau D, Kübler J, Lozajic M, Gabler F, Söding J, Lupas AN, Alva V. 2018. A completely reimplemented MPI bioinformatics toolkit with a new HHpred server at its core. Journal of Molecular Biology 430: 2237-2243. 


\section{Figures}

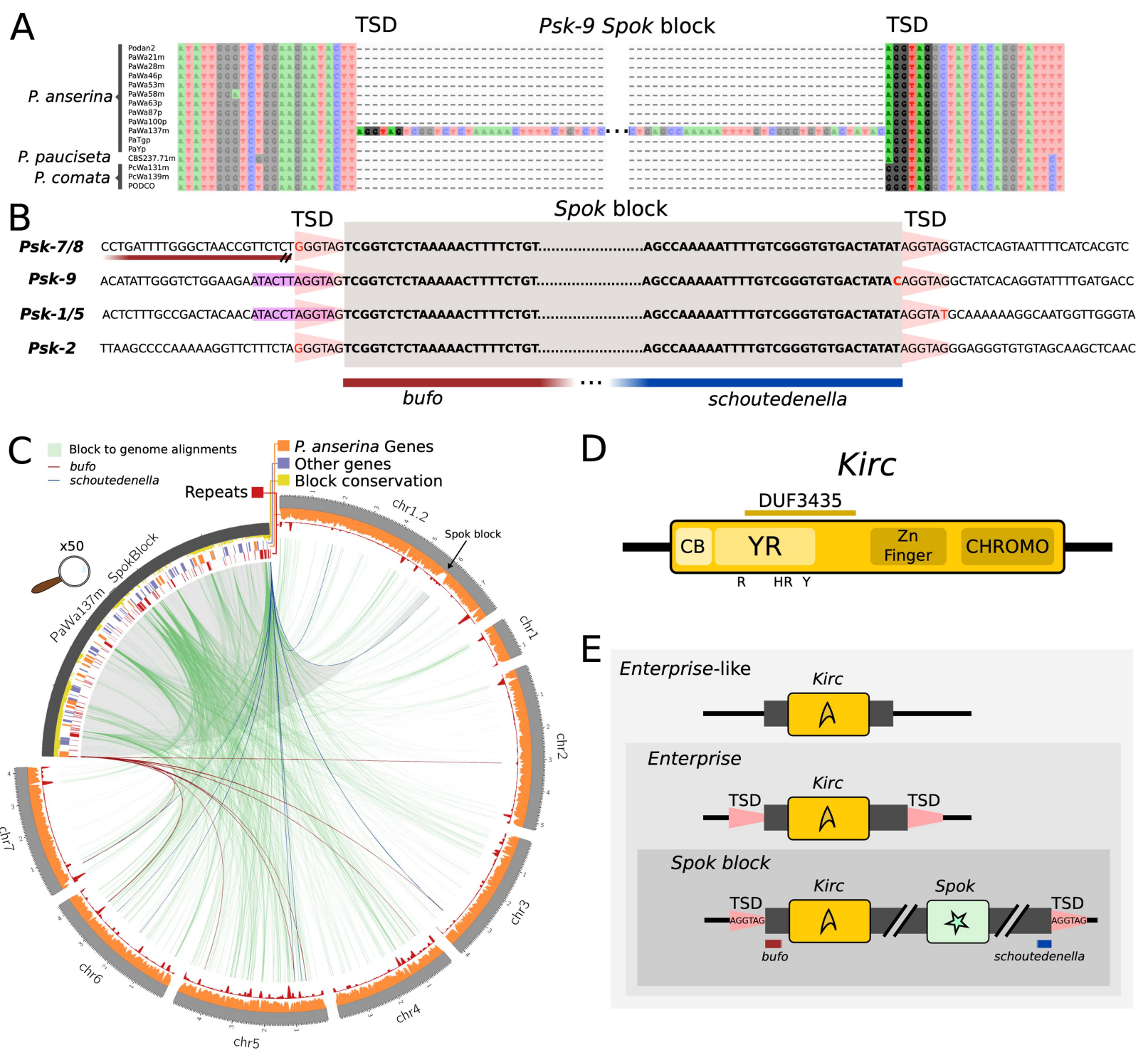

Figure 1. The genomic context of the Spok Block. A An alignment of the region where the Spok block is located in the strain Wa137 (Psk-9) from all $P$. anserina genomes. Putative target site duplication (TSD) is highlighted. Only the ends of the Spok block are shown for clarity. B An alignment of the ends of four versions of the Spok block displaying the TSD (pink trapezoid). The flanks of the Spok block that relate to the unclassified repeats bufo and schoutedenella are underlined in red and blue, respectively. Note that for Psk-7, the Spok block is inserted immediately next to a truncated bufo element. C A Circos plot of the genome of Wa137 (light grey track, size in Mb) aligned against its own Spok block (dark grey, enlarged by 50x). The tracks on the Spok block from outside inward represent: the conservation of regions among the different iterations of the Spok block where the height of the conservation track is equivalent to 
804 the number of blocks that have a given position (yellow); gene models from manual annotation

805 of the Spok block, where genes with homologs within the reference genome of $P$. anserina are

806 marked in orange and those without homologs are marked in purple; and annotated repeated

807 elements (red). The tracks on the chromosomal scaffolds show the coverage of genic regions

808 (orange) and repeats (red) calculated in sliding windows of $50 \mathrm{~kb}$ with steps of $10 \mathrm{~kb}$. Green

809 lines connect homologous segments based on MUMmer alignments. The unclassified repetitive

810 elements bufo and schoutedenella are connected in dark red and blue segments, respectively,

811 based on BLASTn searches. D Cartoon representation of the predicted protein for the ORF Kirc

812 with domains predicted by bioinformatics analyes. The catalytic tetrad is marked below the

813 protein model. E Cartoon model of the hierarchical nomenclature of Enterprise-like elements,

814 Enterprise and the Spok block. See Supplementary Figure 3 for more detailed depictions of the

815 Spok block. 


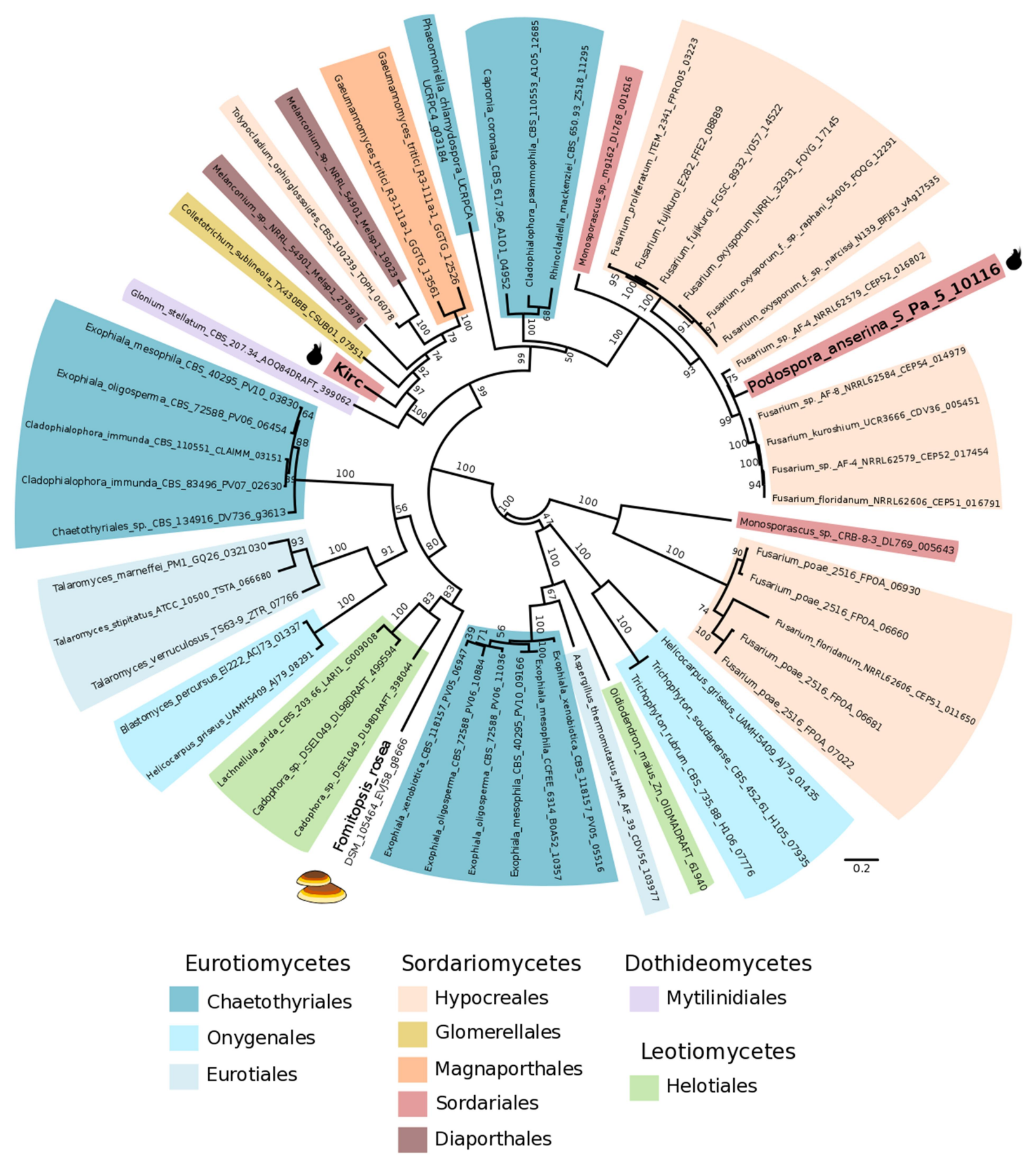

Figure 2. Unrooted Maximum likelihood phylogenetic tree showing the relationship between Kirc and other homologs from fungi. Bootstrap support values are shown above branches.

819 Branch lengths are proportional to the scale bar (amino acid substitution per site). Taxonomic

820 rank is indicated with coloured highlights. The sequences present in Podospora anserina and in

821 the basidiomycete Fomitopsis rosea are marked with cartoons of the corresponding fruiting

822 bodies. Nomenclature is formatted as species_strain_protein code. 


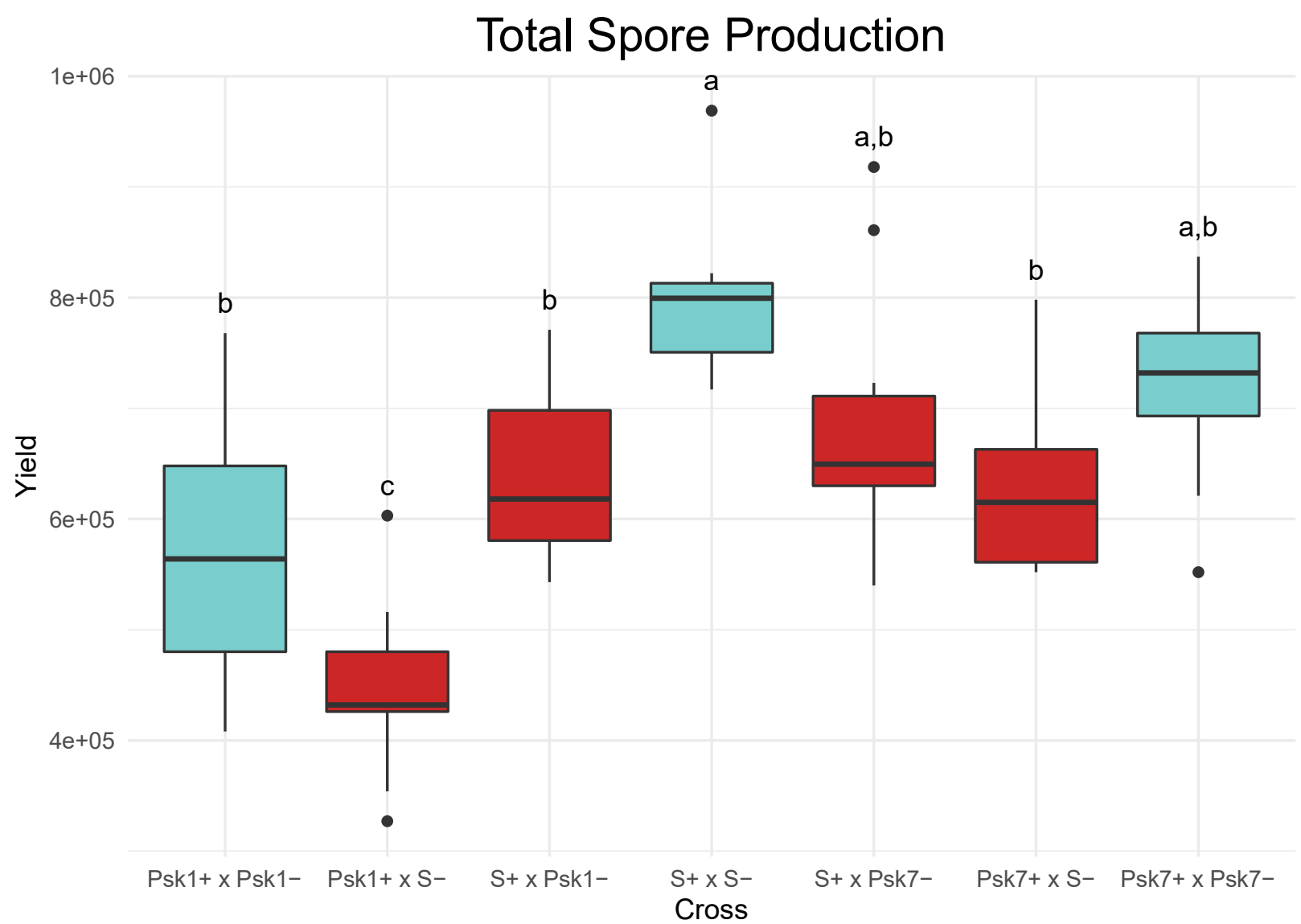

Figure 3. Plot showing the total amount of ascospores collected from crosses of isogenic strains possessing various iterations of the Spok block. Yield obtained in crosses where spore killing occurs are shown in blue; crosses with no spore killing are in red. Letters mark results of a Tukeys HSD test. The mating type is indicated by a + or - after the strain name. The mat+ parent was always used as the maternal strain in crosses. 


\section{Tables}

832 Table 1. List of strains used in this study.

\begin{tabular}{|c|c|c|c|c|c|c|}
\hline Strain & Species & Psk $^{\mathrm{a}}$ & $\begin{array}{l}\text { Genomic } \\
\text { location }\end{array}$ & Size & Enterprise $^{\mathrm{b}}$ & Spok genes \\
\hline Wa21 & P. anserina & 2 & Chr5 R & 142433 & Spok block & Spok2 Spok3 \\
\hline Wa28 & P. anserina & 2 & Chr5 R & 161297 & Spok block & Spok2 Spok3 \\
\hline Wa46 & P. anserina & naïve & - & - & absent & - \\
\hline Wa53 & P. anserina & 1 & Chr 3 & 113407 & Spok block & Spok2 Spok3 Spok4 \\
\hline Wa58 & P. anserina & 7 & Chr $5 \mathrm{~L}$ & 167459 & Spok block & Spok2 Spok3 Spok4 \\
\hline Wa63 & P. anserina & $S$ & - & - & absent & Spok2 \\
\hline Wa87 & P. anserina & 1 & Chr 3 & 113425 & Spok block & Spok2 Spok3 Spok4 \\
\hline Wa100 & P. anserina & 8 & Chr $5 \mathrm{~L}$ & 127869 & Spok block & Spok2 Spok4 \\
\hline Wa137 & P. anserina & 9 & Chr 1 & 247510 & Spok block & Spok2 Spok4 \\
\hline$S$ & P. anserina & $S$ & - & - & absent & Spok2 \\
\hline 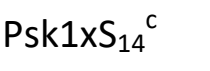 & P. anserina & 1 & Chr 3 & 113407 & Spok block & Spok2 Spok3 Spok4 \\
\hline Psk7xS $_{14}{ }^{c}$ & P. anserina & 7 & Chr $5 \mathrm{~L}$ & 167459 & Spok block & Spok2 Spok3 Spok4 \\
\hline $\mathrm{T}_{\mathrm{G}}$ & P. anserina & 5 & Chr 3 & 157028 & Spok block & Spok3 Spok4 \\
\hline Y & P. anserina & 5 & Chr 3 & 113435 & Spok block & Spok3 Spok4 \\
\hline Wa131 & P. comata & $\mathrm{C} 1$ & - & - & absent & Spok1 \\
\hline Wa139 & P. comata & $\mathrm{C} 1$ & Chr5 L & 38521 & degraded & Spok1 \\
\hline $\mathrm{T}_{\mathrm{D}}$ & P. comata & $\mathrm{C} 1$ & - & - & absent & Spok1 \\
\hline CBS 237.71 & P. pauciseta & P1 & Chr 4 & 74163 & Spok block & Spok3 Spok4 \\
\hline
\end{tabular}

${ }^{a}$ Notice that a given Spok block might have Spok3, Spok4 or both, and this reflects the Psk designation.

${ }^{\text {b}}$ The location of the Spok block and Enterprise element of Wa139 are indicated in the "Genomic Location" column. Spok1 and Spok2 are not found within the Spok block.

' Lab strains created by 14 consecutive backcrosses of Psk-1 or Psk-7 strains to S. The size of the Spok block is assumed to match that of the parental strain. 


\section{Supplementary Figures}

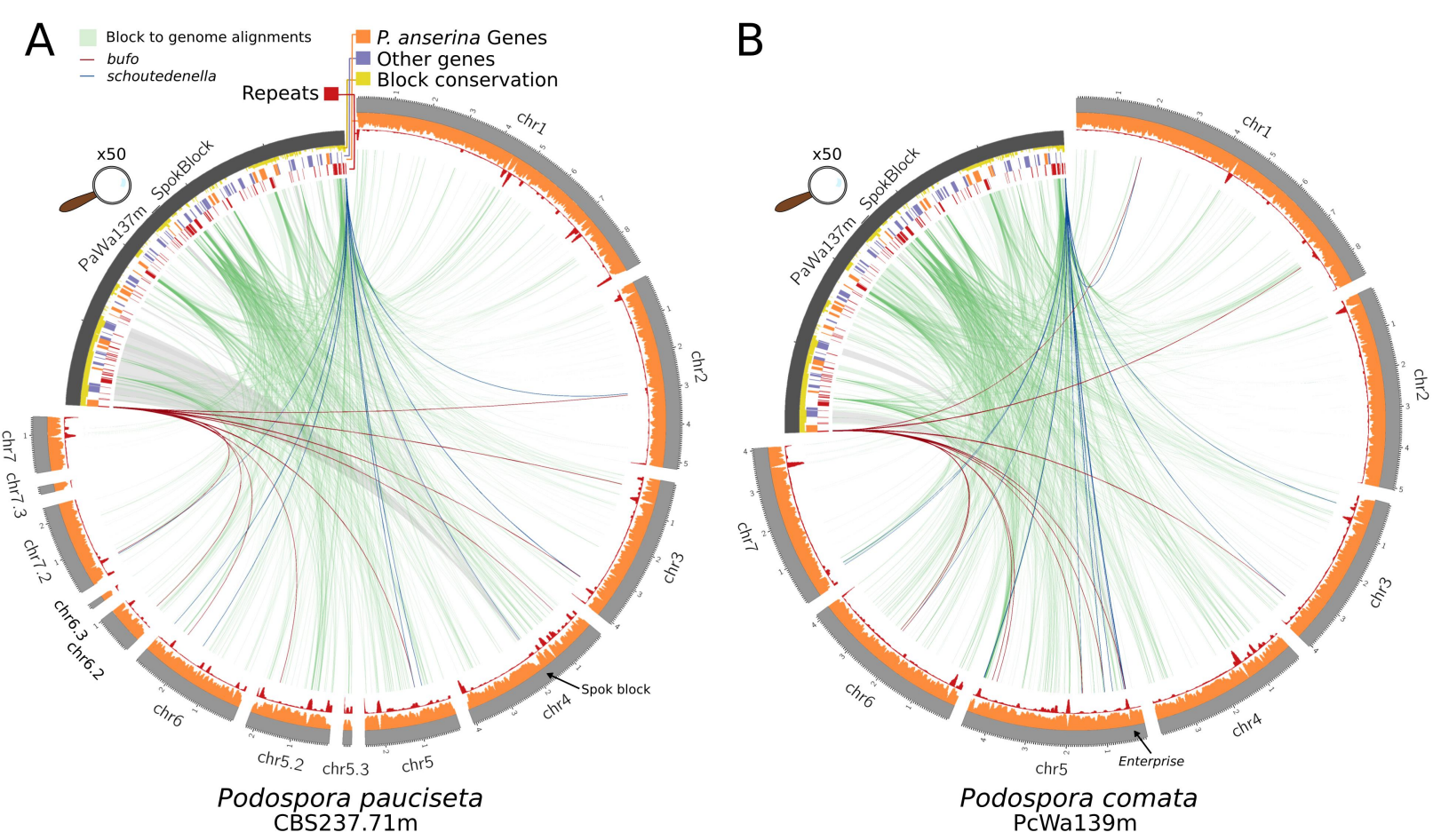

837 the $P$. pauciseta strain CBS237.71 and B the P. comata strain Wa139. 


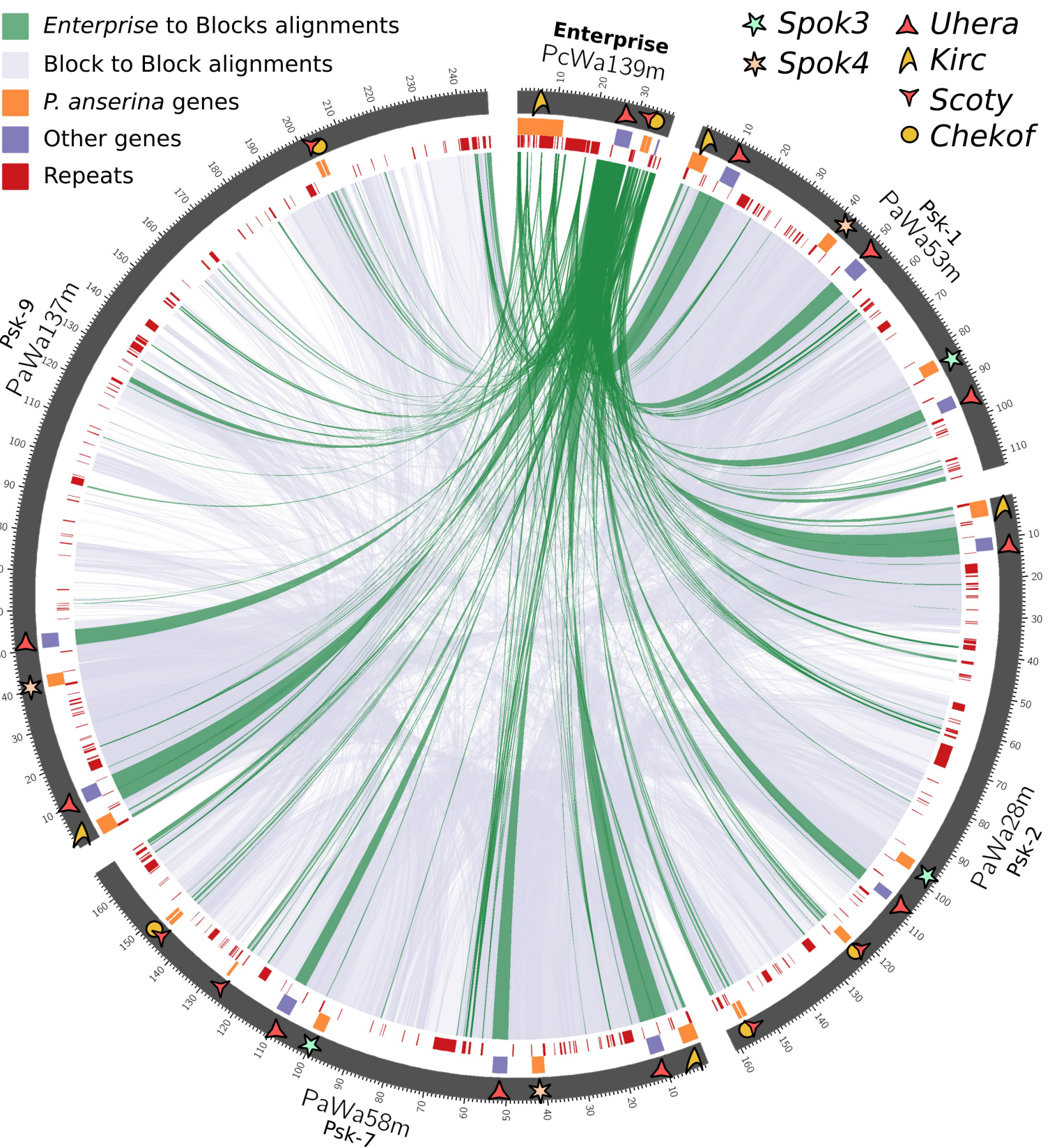

Supplementary Figure 2. A circos plot comparing the Enterprise of five different strains. Four of these represent different Spok blocks. Dark green lines connect homologous segments of the Wa139 Enterprise to the various Spok blocks. Lilac lines show homologous regions among the Spok blocks. Genes of interest are marked with symbols (See Supplementary Table 3). 


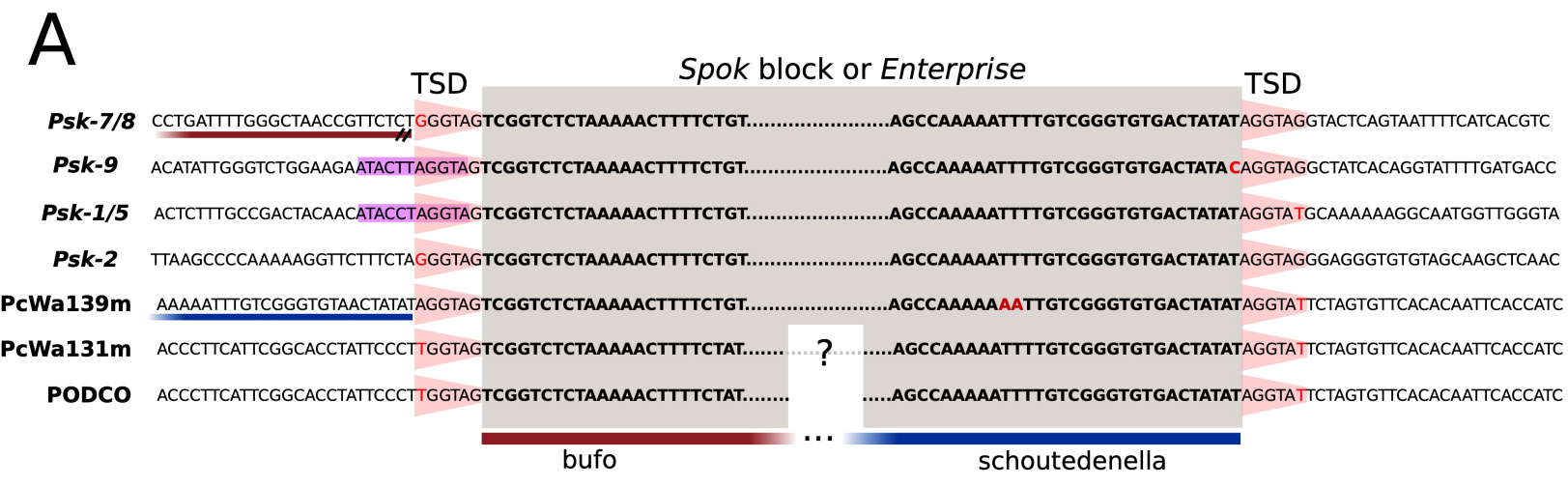

B Spok block
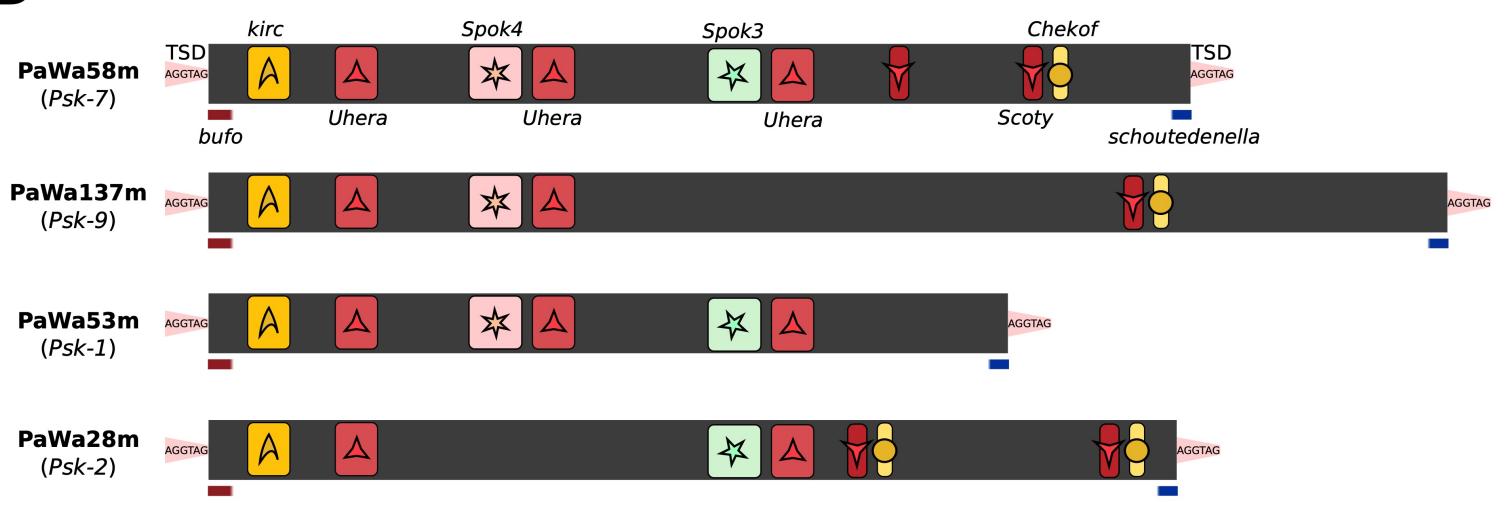

Enterprise

PcWa139m

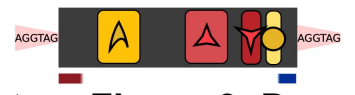

845 Supplementary Figure 3. Description of the Spok Block. A An alignment of the ends of four

846 versions of the Spok block displaying the TSD (red trapezoid) plus the insertion site of

847 Enterprise in three $P$. comata strains. The majority of Enterprise is deleted in the strain $T_{D}$

848 (PODCO) and unassembled in Wa131. Additionally, Wa139 is inserted next to a copy of

849 schoutedenella that is absent in both PODCO and Wa131. B Cartoon models representing the

850 structure and gene content of four Spok blocks and of Enterprise from Wa139. Relevant genes

851 and features are annotated. Not to scale. 


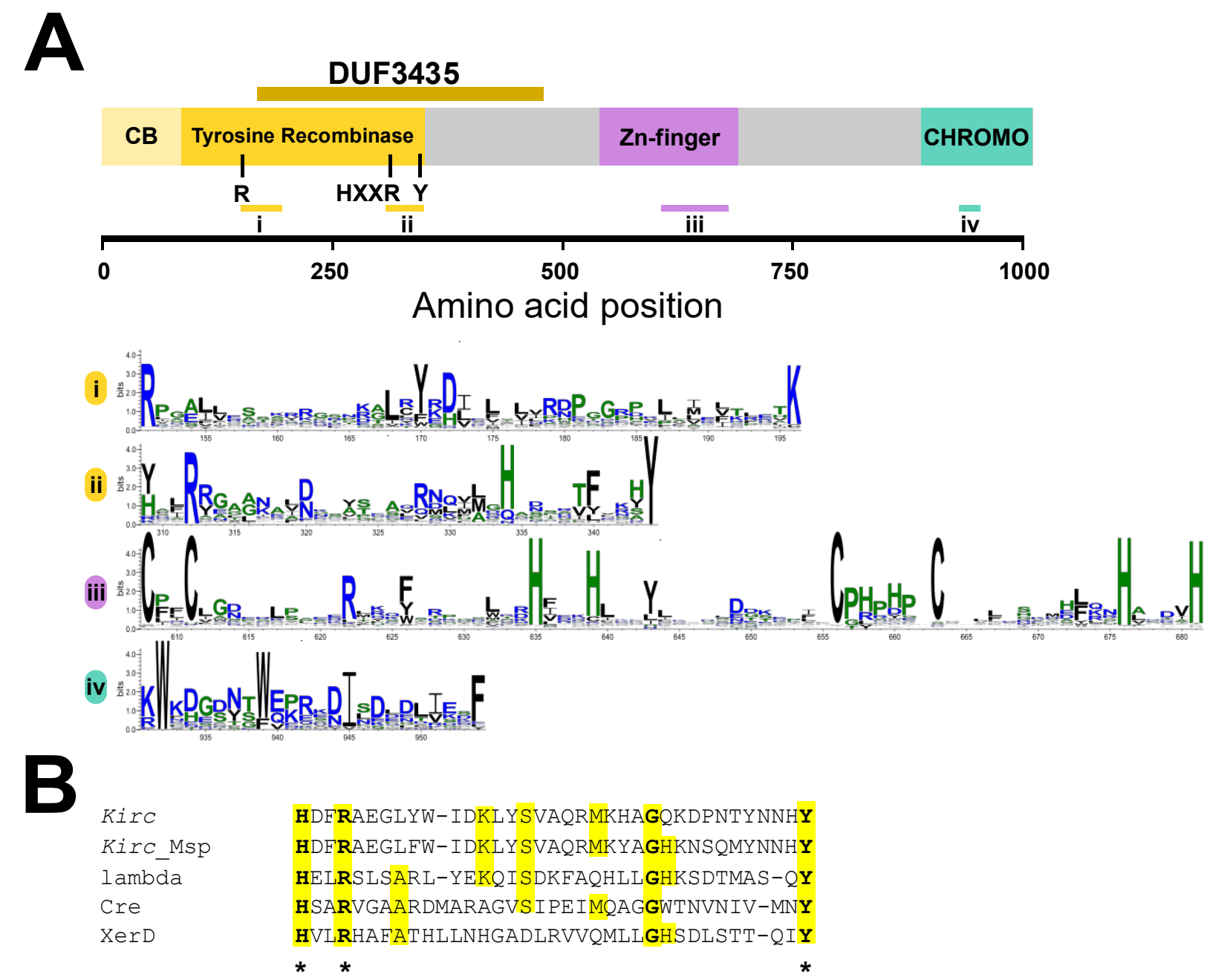

Supplementary Figure 4. Domain annotation of the Kirc protein obtained using HHPred. Four domains were identified: a N-terminal tyrosine recombinase domain with the associated corebinding domain, a central Zn-finger domain and a C-terminal chromodomain. An alignment of 279 Kirc homologs generated by Gremlin with HHBlits was used to create sequence logo of relevant regions of the Kirc sequences, including the active site regions of the tyrosine recombinase domain (i and ii), the $\mathrm{Zn}$-finger region (iii) and the chromodomain region (iv) using Weblogo. B. Alignment of the Kirc sequence with an active site region of known tyrosine recombinases together with the sequence of a Kirc homolog from Melanconium sp.. Alignment is based on the HHPred output. Active site residues are marked with an asterisk. Kirc: Kirc gene in the Spok block of strain Wa58 (sites 309-344); Kirc_Msp: CE55503_10527 (110-145); lambda: lambda integrase PDB: 5J0N (308-342); Cre: recombinase enterobacteriaphage P1, PDB: 1 XO0 (270-305); XerD: XerD site-specific recombinase E. coli, PDB: 1A0P (242-277). 
bioRxiv preprint doi: https://doi.org/10.1101/2020.03.25.007153; this version posted April 28, 2020. The copyright holder for this preprint (which was not certified by peer review) is the author/funder, who has granted bioRxiv a license to display the preprint in perpetuity. It is made available under aCC-BY-NC-ND 4.0 International license.

TSD

Melsp1_01 TCGCATATAGCTACCTACTTAGGTAGTCTTCCGCGGAATCGCACCA.....................AATATGAGGGACGATTATACAGGTAGTCTTATATCGATTTCTGCCC

Melsp1_07 TTCTGCAAACTACCTCCCTAGGTAGTCTTCCGCGGAATCGCACCA.....................AATATGAGGGACGACTATACAGGTAGTATCGGTGACTCAGAAGCT Melsp1_11 AATAGCTATATAAACTACTTAGGTAGTCTTCCGCGGAATCGCACCA....................AATACGAGgGACGACTATACAGGTAATTATCTGGATTAATAATCAT

Melsp1_13 TIGTCTCCGTGGACCTACCTAGGTAGTCTTCCGCAGAATCACACCA...................AATATGAGGGATAACTATACAGGTATGGTTGTGGGGCTTCTTAGA

868 Supplementary Figure 5. Alignment of four Enterprise elements within Melanconnium sp.

869 revealing the TSD. 
Supplementary Table 1. Assembly statistics for all strains with genomic data used in this study.

\begin{tabular}{|c|c|c|c|c|c|c|c|c|c|}
\hline Species & Strain & Assembly & Technology & Assembler & Scaffolds $^{a}$ & N50 & GC content & Size (bp) & $\begin{array}{c}\text { Mean Depth } \\
\text { long (x) }\end{array}$ \\
\hline P. anserina & Wa21- & PaWa21m & PacBio & HGAP 3.0 & 9 & 5072363 & 51.49 & 35767411 & 80.3071 \\
\hline P. anserina & Wa28- & PaWa28m & PacBio & HGAP 3.0 & 7 & 5076631 & 51.41 & 35929333 & 76.2045 \\
\hline P. anserina & Wa46+ & PaWa46p & PacBio & HGAP 3.0 & 9 & 4338573 & 51.37 & 35724019 & 117.8295 \\
\hline P. anserina & Wa53- & PaWa53m & PacBio & HGAP 3.0 & 7 & 4924837 & 51.52 & 35808992 & 83.821 \\
\hline P. anserina & Wa58- & PaWa58m & PacBio & HGAP 3.0 & 7 & 5037662 & 51.46 & 35985748 & 112.1709 \\
\hline P. anserina & Wa63+ & PaWa63p & PacBio & HGAP 3.0 & 7 & 4907271 & 51.36 & 36002190 & 111.2245 \\
\hline P. anserina & Wa87+ & PaWa87p & PacBio & HGAP 3.0 & 9 & 4338609 & 51.44 & 35896992 & 105.8792 \\
\hline P. anserina & Wa100+ & PaWa100p & PacBio & HGAP 3.0 & 7 & 5096663 & 51.5 & 35953417 & 114.091 \\
\hline P. anserina & Wa137- & PaWa137m & MinION & Miniasm 0.2 & 9 & 4341106 & 51.08 & 36531367 & 49.924 \\
\hline P. anserina & $\mathrm{Y}+$ & PaYp & MinION & Miniasm 0.2 & 8 & 4821330 & 51.55 & 35719817 & 35.6112 \\
\hline P. anserina & $\mathrm{T}_{\mathrm{G}^{+}}$ & PaTgp & MinION & Miniasm 0.2 & 13 & 4230402 & 51.54 & 35778794 & 37.6743 \\
\hline P. pauciseta & CBS237.71- & CBS237.71m & MinION & Miniasm 0.2 & 13 & 4070182 & 51.6 & 35576233 & 24.3067 \\
\hline P. comata & Wa131- & PcWa131m & Illumina HiSeq & SPAdes 3.12 .0 & 1119 & 258199 & 52.1 & 34680523 & - \\
\hline P. comata & Wa139- & PcWa139m & MinION & Miniasm 0.2 & 7 & 4603841 & 51.98 & 34819024 & 52.5779 \\
\hline P. anserina & S+ & Podan2 & WGS & Arachne & 7 & 4734292 & 52.17 & 35010595 & - \\
\hline P. comata & $\mathrm{T}_{\mathrm{D}^{+}}$ & PODCO & 454 + Illumina & Newbler 2.3 & 7 & 4662433 & 52.42 & 34385490 & - \\
\hline
\end{tabular}

${ }^{a}$ The number of scaffolds correspond to those that map to the 7 chromosomes (excluding mitochondrial and rDNA contigs), but other columns include all scaffolds

${ }^{\mathrm{b}}$ Numbers correspond to long-read data when available, otherwise to short-read data. For Wa139- and Wa137- the reads were filteread for QV $>9$ and to be larger than $1 \mathrm{~kb}$, resulting in 184301 and 202279 reads, respectively.

'Scaffolds larger than $50 \mathrm{~kb}$, excluding the mitochondrial and rDNA scaffolds 


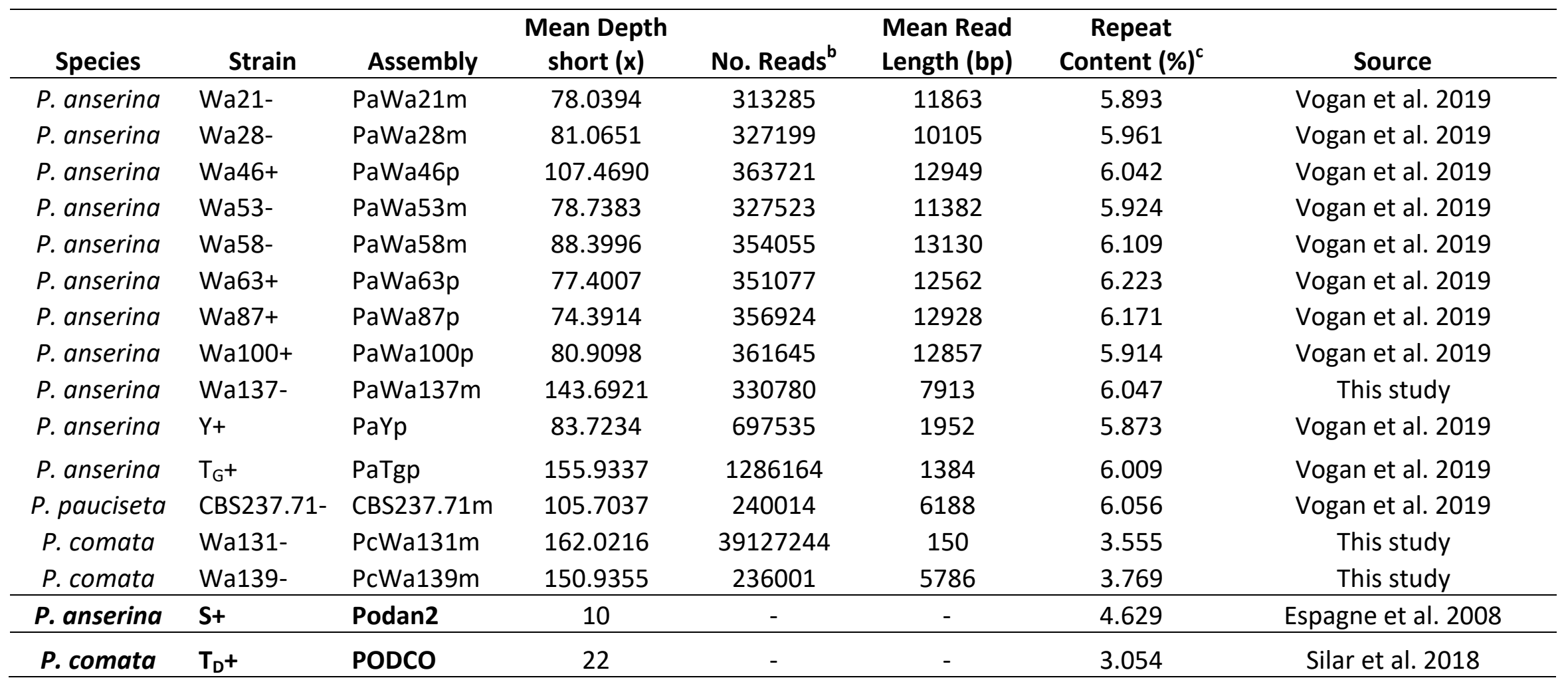


Supplementary Table 2. Hypothetical genes from within the Spok block of Wa137. Pfam domains inferred from NCBI conserved domain database. Putative functions inferred from BLASTp to other genes with functional annotations. Blank cells indicate either no pfam hits, or genes with BLASTp results to only hypothetical genes.

876

\begin{tabular}{|c|c|c|c|c|}
\hline \multirow{2}{*}{$\begin{array}{l}\text { Gene code / name in Wa137 } \\
\text { gene } 01805 \text { | Kirc }\end{array}$} & \multicolumn{3}{|c|}{ pfam } & \multirow[t]{2}{*}{ Putative function } \\
\hline & $\begin{array}{l}\text { DUF3435 } \\
\text { superfamily }\end{array}$ & $\begin{array}{l}\text { CD_CSD } \\
\text { superfamily }\end{array}$ & CHROMO & \\
\hline gene01806|Uhera1 & $\begin{array}{l}\text { DEAD-like_- } \\
\text { helicase_C } \\
\text { superfamily }\end{array}$ & $\begin{array}{l}\text { DEAD- } \\
\text { like_helicase_N } \\
\text { superfamily }\end{array}$ & & \\
\hline $\begin{array}{l}\text { PaWa137m.02400|PpBr36_10652_ } \\
\text { Pyricularia_pennisetigena }\end{array}$ & $\begin{array}{l}\text { Peptidase_C48 } \\
\text { superfamily }\end{array}$ & & & \\
\hline $\begin{array}{l}\text { PaWa137m.02401| } \\
\text { Pa_2_5490_homolog }\end{array}$ & NAP & & & \\
\hline $\begin{array}{l}\text { PaWa137m.02402|S7711_11618_ } \\
\text { Stachybotrys_chartarum_homolog }\end{array}$ & & & & $\begin{array}{l}\text { NAD-dependent protein } \\
\text { deacetylase }\end{array}$ \\
\hline \multicolumn{5}{|l|}{$\begin{array}{l}\text { PaWa137m_chromosome_1.2:hsp:12095 } \\
\text { 1:4.5.0.58|Pa_7_10380_fragment }\end{array}$} \\
\hline $\begin{array}{l}\text { PaWa137m_CH35J_006576| } \\
\text { CH35J_006576_Colletotrichum_ } \\
\text { higginsianum_fragment }\end{array}$ & $\begin{array}{l}\text { Patatin_and_cPLA2 } \\
\text { superfamily }\end{array}$ & & & Phospholipase A2 \\
\hline $\begin{array}{l}\text { PaWa137m.02404| } \\
\text { PODCO_502086_Fragment }\end{array}$ & $\begin{array}{l}\text { DDE_Tnp_ISL3 } \\
\text { superfamily }\end{array}$ & & & PKS-NRPS hybrid synthetase \\
\hline \multicolumn{5}{|l|}{ PaWa137m.02405| Pa_4_1058_homolog } \\
\hline gene00315|Spok4 & & & & Spore killer \\
\hline $\begin{array}{l}\text { PaWa137m.Pa_5_2010_fragment1| } \\
\text { Pa_5_2010_fragment1 }\end{array}$ & & & & $\begin{array}{l}\text { GPI anchored serine-threonine } \\
\text { rich protein }\end{array}$ \\
\hline \multicolumn{5}{|l|}{ gene01852|Uhera2 } \\
\hline $\begin{array}{l}\text { PaWa137m_Pa_1_14730_fragment1 | } \\
\text { Pa_1_14730_fragment }\end{array}$ & & & & EST/SMG-like protein 1 \\
\hline
\end{tabular}




\begin{tabular}{|c|c|c|c|c|c|}
\hline gene01815|Pa_6_9560_Fragment & p450 superfamily & & & & pisatin demethylase \\
\hline gene01816|Pa_6_9560_homolog & p450 superfamily & & & & pisatin demethylase \\
\hline $\begin{array}{l}\text { PaWa137m_7_6640_fragment | } \\
\text { Pa_7_6640_Fragment }\end{array}$ & $\begin{array}{l}\text { Peptidase_M16_M } \\
\text { superfamily }\end{array}$ & & & & insulysin \\
\hline $\begin{array}{l}\text { PaWa137m_CTA1_5148| } \\
\text { PaWa137m_CTA1_5148_- } \\
\text { Colletotrichum_tanaceti_fragment }\end{array}$ & NK superfamily & & & & Uridylate kinase \\
\hline $\begin{array}{l}\text { PaWa137m.gene.002245| } \\
\text { Pa_6_9580_homolog }\end{array}$ & & & & & $\begin{array}{l}\text { Satratoxin biosynthesis SC1 } \\
\text { cluster protein } 4\end{array}$ \\
\hline gene01818|Pa_4_3510_homolog & $\begin{array}{l}\text { Peptidase_C48 } \\
\text { superfamily }\end{array}$ & $\begin{array}{l}\text { GumC } \\
\text { superfamily }\end{array}$ & & & \\
\hline $\begin{array}{l}\text { PaWa137m.gene.002249| } \\
\text { Pa_1_13070_fragment }\end{array}$ & $\mathrm{zf}-\mathrm{H} 2 \mathrm{C} 2 \_2$ & $\mathrm{zf}-\mathrm{C} 2 \mathrm{H} 2$ & COG5048 & & cutinase G-box binding protein \\
\hline $\begin{array}{l}\text { PaWa137m.gene.PODCO_102140| } \\
\text { PODCO_102140_fragment }\end{array}$ & $\begin{array}{l}\text { ABC_ATPase } \\
\text { superfamily }\end{array}$ & & & & Heavy metal tolerance protein \\
\hline $\begin{array}{l}\text { gene01820|PV05_03319_ } \\
\text { Exophiala_xenobiotica_fragment }\end{array}$ & MdIB & & & & Heavy metal tolerance protein \\
\hline \multicolumn{6}{|c|}{ PaWa137m.02410| Pa_6_7965_homolog } \\
\hline gene01823|Pa_1_13070_homolog & $\mathrm{zf}-\mathrm{C} 2 \mathrm{H} 2$ & $\mathrm{zf}-\mathrm{H} 2 \mathrm{C} 2 \_2$ & $\mathrm{zf}-\mathrm{C} 2 \mathrm{H} 2$ & $\begin{array}{l}\text { lambda-1 } \\
\text { superfamily }\end{array}$ & cutinase G-box binding protein \\
\hline \multicolumn{6}{|l|}{$\begin{array}{l}\text { PaWa137m_Pa_6_2490_fragment } \\
\text { Pa_6_2490_fragment }\end{array}$} \\
\hline \multicolumn{6}{|l|}{ gene01824| PODCO_511675_homolog } \\
\hline $\begin{array}{l}\text { PaWa137m_Pa_6_2440_fragment| } \\
\text { Pa_6_2440_fragment }\end{array}$ & $\begin{array}{l}\text { PRK09605 } \\
\text { superfamily }\end{array}$ & & & & \\
\hline $\begin{array}{l}\text { PaWa137m_MBR_09427_ } \\
\text { Metarhizium_brunneum_fragment } \\
\text { PaWa137m_MBR_09427_ } \\
\text { Metarhizium_brunneum_fragment }\end{array}$ & & & & & mitofusin \\
\hline $\begin{array}{l}\text { gene01824.2| } \\
\text { PODCO_511675_homolog_2 }\end{array}$ & & & & & \\
\hline
\end{tabular}




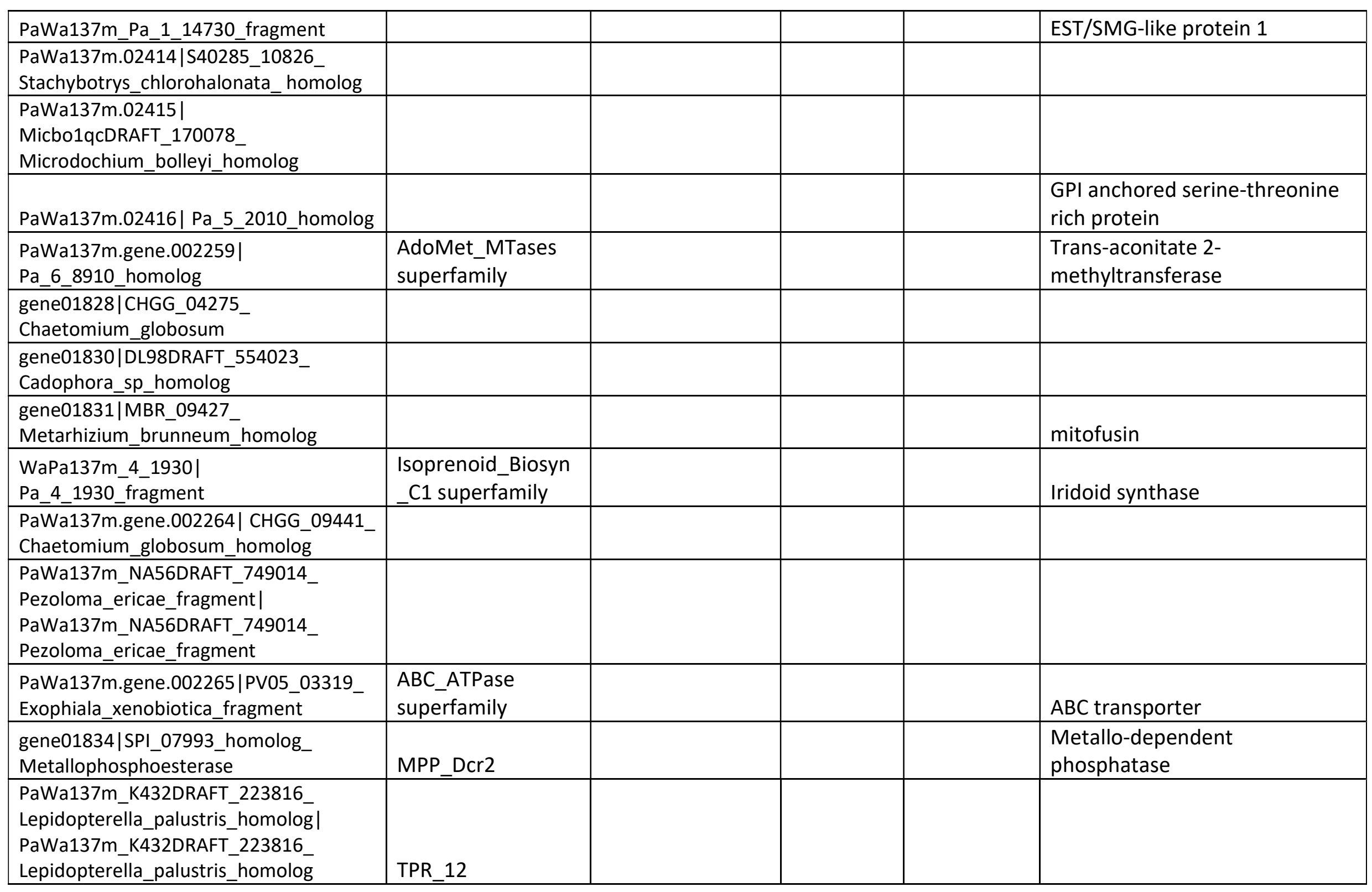




\begin{tabular}{|c|c|c|c|}
\hline gene01835|Pa_1_1830_homolog & GT2_HAS & & Hyaluronan synthase \\
\hline gene01836|Pa_1_14730_homolog & $\begin{array}{l}\text { EST1_DNA_bind } \\
\text { superfamily }\end{array}$ & & EST/SMG-like protein 1 \\
\hline $\begin{array}{l}\text { PaWa137m.02425|S7711_10142_- } \\
\text { Stachybotrys_chartarum_homolog }\end{array}$ & $\begin{array}{l}\text { CD_CSD } \\
\text { superfamily }\end{array}$ & & \\
\hline $\begin{array}{l}\text { PaWa137m_HIM_12183|HIM_12183_ } \\
\text { Hirsutella_minnesotensis_homolog }\end{array}$ & \begin{tabular}{|l} 
Pkinase \\
superfamily
\end{tabular} & & \\
\hline \multicolumn{4}{|l|}{$\begin{array}{l}\text { PaWa137m_M441DRAFT_90628| } \\
\text { M441DRAFT_90628_ } \\
\text { Trichoderma_asperellum_homolog }\end{array}$} \\
\hline \multicolumn{4}{|l|}{$\begin{array}{l}\text { gene01838|S7711_11475__ } \\
\text { Stachybotrys_chartarum_homolog }\end{array}$} \\
\hline $\begin{array}{l}\text { gene01839|S40293_11413_ } \\
\text { Stachybotrys_chartarum }\end{array}$ & & & patatin-like serine hydrolase \\
\hline $\begin{array}{l}\text { gene01840|NA56DRAFT_703776_ } \\
\text { Pezoloma_ericae_homolog }\end{array}$ & \begin{tabular}{|l|} 
DUF3723 \\
superfamily \\
\end{tabular} & $\begin{array}{l}\text { PTZ00121 } \\
\text { superfamily }\end{array}$ & \\
\hline $\begin{array}{l}\text { gene01841|Micbo1qcDRAFT_129033_ } \\
\text { Microdochium_bolleyi_homolog }\end{array}$ & & & $\begin{array}{l}\text { Satratoxin biosynthesis SC1 } \\
\text { cluster protein } 4\end{array}$ \\
\hline gene01842|Pa_5_3190_homolog & MFS superfamily & & Efflux pump FUS6 \\
\hline gene01843|Pa_7_6640_homolog & Ptr superfamily & & insulysin \\
\hline \multicolumn{4}{|l|}{$\begin{array}{l}\text { PaWa137m_SS1G_08066_Sclerotinia_ } \\
\text { sclerotiorum_homolog|Scoty }\end{array}$} \\
\hline gene01844|Chekof & $\begin{array}{l}\text { PKc_like } \\
\text { superfamily }\end{array}$ & & \\
\hline PaWa137m_Pa_1_1830_fragment & \begin{tabular}{|l}
$\begin{array}{l}\text { Peptidase_C48 } \\
\text { superfamily }\end{array}$ \\
\end{tabular} & & \\
\hline \multicolumn{4}{|l|}{$\begin{array}{l}\text { PaWa137m.gene.002278|PpBr36_10652 } \\
\text { _Pyricularia_pennisetigena_homolog }\end{array}$} \\
\hline \multicolumn{4}{|l|}{ gene01845|Pa_4_3510_fragment } \\
\hline $\begin{array}{l}\text { gene01847|GE09DRAFT_1263105_ } \\
\text { Coniochaeta_sp_homolog }\end{array}$ & MdlB & & Heavy metal tolerance protein \\
\hline
\end{tabular}




\begin{tabular}{|c|c|c|}
\hline $\begin{array}{l}\text { gene01849|CHGG_09572_- } \\
\text { Chaetomium_globosum_fragment }\end{array}$ & & Putative AC transposase \\
\hline $\begin{array}{l}\text { gene01850|MBR_09427_ } \\
\text { Metarhizium_brunneum_homolog }\end{array}$ & & \\
\hline $\begin{array}{l}\text { PaWa137m_AJ78_06594_- } \\
\text { Emergomyces_pasteurianus_homolog } \\
\text { PaWa137m_AJ78_06594__ } \\
\text { Emergomyces_pasteurianus_homolog }\end{array}$ & & \\
\hline PaWa137m.02432|Pa_5_3190_homolog & MFS superfamily & Efflux pump FUS6 \\
\hline $\begin{array}{l}\text { PaWa137m_CSUB01_08519_- } \\
\text { Colletotrichum_sublineola_homolog }\end{array}$ & CFEM superfamily & $\begin{array}{l}\text { Satratoxin biosynthesis SC1 } \\
\text { cluster protein } 4\end{array}$ \\
\hline $\begin{array}{l}\text { PaWa137m.gene.002286| } \\
\text { MYCTH_64320_ } \\
\text { Thermothelomyces_thermophilus_ } \\
\text { homolog }\end{array}$ & & \\
\hline $\begin{array}{l}\text { PaWa137m_DL765_007700_ } \\
\text { Monosporascus_sp_fragment| } \\
\text { PaWa137m_DL765_007700_ } \\
\text { Monosporascus_sp_fragment }\end{array}$ & & \\
\hline
\end{tabular}

